Ann. Biol. anim. Bioch. Biophys., 1979, 19 (1 A), 1-30.

\title{
Etude morpho-anatomique du périmysium (Méthodes de des- cription et d'évaluation)
}

\author{
par O. SCHMITT, Thérèse DEGAS, P. PEROT, Marie-Reine LANGLOIS, \\ B. L. DUMONT \\ Laboratoire de recherches sur la Viande I. N. R. A., 78350 Jouy-en-Josas.
}

Summary. A morphological study of skeletal muscle perimysium. Methods of description and appraisal.

An analysis of the organization of the perimysium of skeletal muscle is presented. It is based on a detailed histological study of the cross-sections of a large number of various muscles from different species of mammals. Two parts of the perimysium are distinguished :

- a major connective tissue network defining muscle texture and easily distinguishable either macroscopically or at low magnification ; cation.

- a secondary network which is only visible as a whole at a rather high magnifi-

The organization of both connective tissue networks were defined by considering various morphological traits, and objective and subjective methods were suggested for their appraisal. The reference scales used were illustrated. The proposed methods would have various useful applications : systematic studies of perimysium of different muscles, influence of biological factors of variation (age, sex, genetics...) and, in the case of meat animals, consequences on meat texture and tenderness.

\section{Introduction}

La trame périmysiale

La trame périmysiale principale

- Définition

- Détermination

La trame périmysiale secondaire

Méthodes de description des caractères définissant la structure musculaire

Matériel et méthodes

Niveau et seuil d'observations

Les caractères observés

Ensemble du périmysium

- Importance du tissu conjonctif périmysial total

- Densité du tissu conjonctif périmysial total 
- Structuration du tissu conjonctif périmysial :

- notion de structuration,

- appréciation du degré de structuration,

- structuration atypique.

Caractères de la trame périmysiole principole

- Caractères généraux :

- uniformité de son épaisseur,

- épaisseur moyenne.

- Les faisceaux principaux :

- nombre de faisceaux principaux,

- forme des faisceaux principaux,

- dimension des faisceaux principaux,

- faisceaux principaux de petite taille,

- relation forme/dimensions des faisceaux principaux.

- Caractères des parois de la trame principale

- uniformité de l'épaisseur,

- forme des sommets,

- les nœuds.

Caractères des trames périmysiales secondaires:

- homogénéité,

- présence d'étoiles conjonctives,

- finesse,

- vaisseaux sanguins, lames conjonctives, taches conjonctives.

\section{Introduction.}

Lorsqu'on considère la structure d'un muscle volontaire il est utile de commencer par examiner la section du muscle dans son entier (Gould, 1973). C'est, en effet, par l'examen de l'ensemble du muscle qu'on peut le mieux appréhender la base de l'organisation de la structure, c'est-à-dire de l'arrangement de groupes de fibres musculaires, par l'intermédiaire du tissu conjonctif qui joue un rôle d'emballage et de séparation (Cassens, 1971). Le cloisonnement du muscle en faisceaux d'inégale importance, connu de longue date (Bourgelat, 1797) est maintenant une notion classique de la myologie. Partant de l'aponévrose qui entoure le muscle et qu'on appelle l'épimysium, des travées de tissu conjonctif pénètrent en profondeur au sein du muscle pour y entourer et y séparer les faisceaux de fibres musculaires individuelles. L'ensemble de la trame d'union des différents faisceaux représente le périmysium. Au sein de chaque faisceau, l'endomysium est le tissu conjonctif intrafasciculaire qui entoure chacune des fibres musculaires (cf. Forrest et al., 1975). Le périmysium est intéressant à considérer à la fois dans ses relations avec le fonctionnement du muscle vivant et, chez l'animal de boucherie, dans ses rapports avec la dureté et, d'une façon, plus générale, avec les propriétés de texture des viandes. On peut supposer que, dans une certaine mesure, la trame du tissu conjonctif doit régler ef contrôler l'importance de la contraction réalisée par le muscle (Gould, 1973). Il est intéressant de noter, par exemple, que la proportion de tissu conjonctif est plus forte dans les muscles qui sont capables de mouvements finement gradués (Fernand, 1949).

Dans le cas des viandes, la trame de tissu conjonctif représente une sorte de char- 
pente interne des morceaux - très variable en importance et dans sa répartition et contribue largement à expliquer les différences de tendreté constatées d'un muscle à l'autre (Dumont, 1952).

Jusqu'à maintenant, à notre connaissance, l'agencement spatial du réseau de tissu conjonctif intramusculaire n'a pas donné lieu à des études systématiques. On n'en connaît ni les caractéristiques, ni les règles d'organisation. Les difficultés techniques d'obtention et d'analyse des échantillons expliquent cette situation, spécialement dans le cas des gros mammifères où les muscles atteignent des dimensions importantes.

Les études réalisées dans notre laboratoire sur l'organisation musculaire des animaux de boucherie nous ont conduits à nous intéresser particulièrement au développement de la trame conjonctive intramusculaire et à ses conséquences. Nous avons été amenés, notamment, à en définir les principales caractéristiques et à tenter d'en apprécier l'importance et la variation. Le présent travail expose les bases de la méthode que nous proposons pour décrire les principaux traits de la trame conjonctive intramusculaire.

\section{La trame périmysiale.}

- Définition. - La trame périmysiale correspond à l'ensemble du tissu conjonctif entourant les faisceaux de fibres musculaires. Sur la base de considérations morpho-anatomiques, nous proposons de la séparer en deux parties :

- une trame périmysiale principale, responsable de la structure macroscopique du muscle ;

- une trame périmysiale secondaire ou complémentaire.

En considérant aftentivement la surface de section d'un muscle à faible grossissement ou même en grandeur nature pour les muscles des gros mammifères, on constate généralement, au sein du périmysium, l'existence d'une trame apparaissant nettement sous la forme d'un réseau caractéristique d'un type de muscle déterminé, à un niveau donné (par rapport aux extrémités du muscle). C'est cette trame que nous appellerons la trame périmysiale principale. Cette trame représente un ensemble de travées conjonctives entrecroisées plus ou moins régulières. Les contours de cette trame délimitent des surfaces d'importance et de forme parfois très variables mais dont l'ensemble a une allure globale propre à chaque muscle. Pour qualifier ces éléments on a proposé (Schmitt et Dumont, 1969) le terme de Myoskhène pour traduire qu'il pourrait s'agir d'unités de la structure musculaire. On peut considérer que ces éléments sont, pour le muscle, des faisceaux principaux, généralement faisceaux tertiaires ou quaternaires qui regroupent un nombre important de faisceaux primaires ef de faisceaux secondaires.

- Détermination. - La détermination du tracé de la trame principale de tissu conjonctif donne évidemment lieu à une appréciation subjective de la part de l'opérateur qui doit apprécier la trame, en tenant compte à la fois de son épaisseur relative par rapport au restant du périmysium qu'elle entoure et en considérant, d'autre part, la régularité de sa répartition. Selon les muscles, la trame conjonctive principale apparaît plus ou moins nettement et sa détermination est plus ou moins aisée. 
Sur le muscle frais en l'état la trame principale de tissu conjonctif se perçoit d'autant plus facilement qu'après la coupe les différences de consistance entre les faisceaux de fibres et la trame entraînent de légères déformations des faisceaux par rapport à celle-ci, ce qui en facilite les délimitations.

Sur muscle fixé et coupé pour examen histologique la trame se distingue par son épaisseur relativement uniforme et par la régularité de sa répartition. II esi fréquent, en effet, qu'au sein du muscle on voit se dessiner un certain nombre de travées conjonctives qui sont orientées selon quelques directions particulières, un peu comme peuvent l'être, dans un réseau routier ou ferroviaire, les lignes principales de communication.

En ce qui concerne l'épaisseur on peut admettre qu'en règle générale les contours de la trame principale sont nettement plus épais que ne le sont les parois des tissus conjonctifs qui déterminent, au sein de la trame, les séparations entre les faisceaux primaires, ou entre les faisceaux secondaires. II peut arriver cependant que la différence d'épaisseur soił faible, voire inexistante. Dans ce cas les caractères d'orientation de la trame peuvent guider l'observateur pour définir les unités de structure.

La figure 1 donne deux illustrations de la détermination du tracé de la trame principale dans le cas de deux types de muscles (a, M. rhomboïdeus; b, M. splenius). $a$ et $b$ représentent les photographies des coupes histologiques sur lesquelles apparaît l'ensemble du périmysium visible au grossissement considéré ; on a renforcé à l'encre, les photographies $a^{\prime}, b^{\prime}$ pour indiquer comment l'observateur avait retenu le tracé de la trame principale.

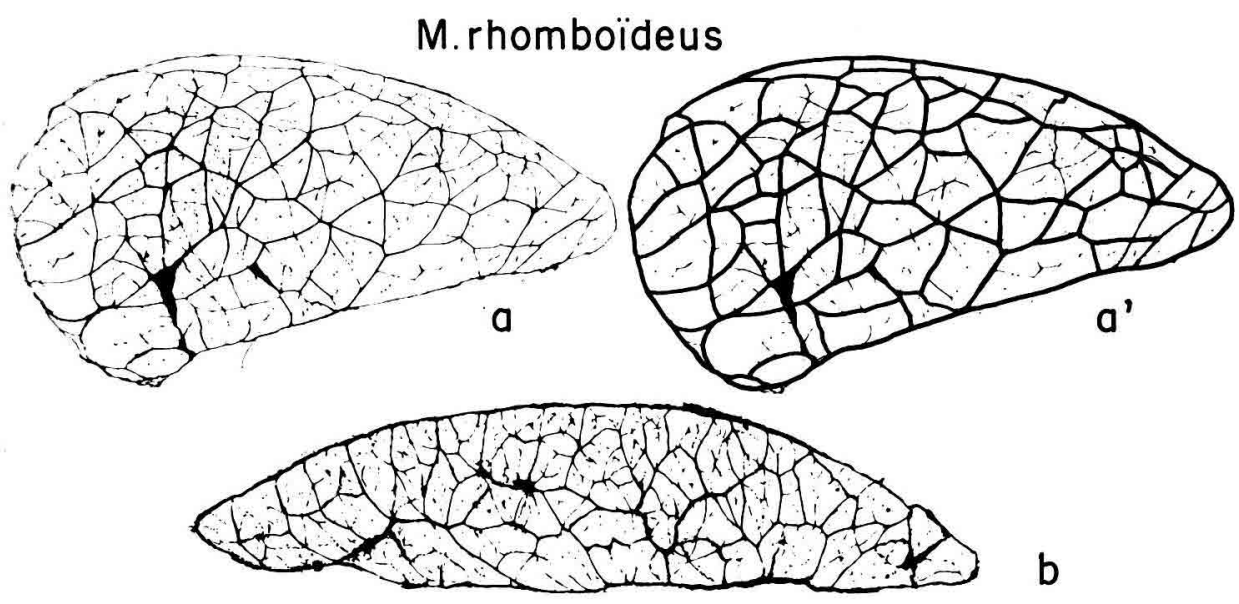

M.splenius

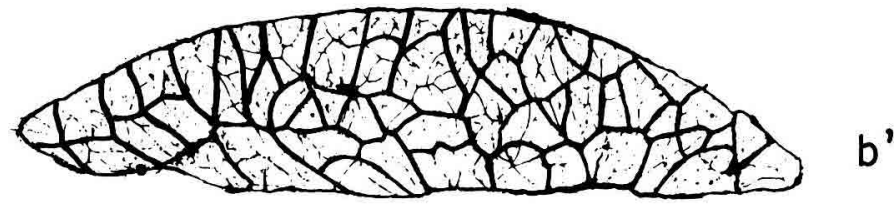

FIG. 1. - Exemple de détermination du tracé de la trame principale $(\times 1)$. 


\section{La trame périmysiale secondaire.}

D'après la hiérarchie que nous avons proposée pour les différentes parties du périmysium on peut parler d'une trame périmysiale principale, et à l'intérieur de chaque faisceau principal d'une trame périmysiale secondaire qui comprend tout le périmysium autre que le principal. Lorsqu'on examine la surface de section d'un muscle à l'œil nu et en grandeur nature, c'est surtout la trame périmysiale principale qu'on est amené à percevoir en premier lieu. Lorsqu'on examine la surface de section d'un muscle à un grossissement de plus en plus fort, on distingue plus difficilement la trame périmysiale principale. A très fort grossissement même, les dimensions du champ

\section{TABLEAU 1A}

Table de description de la frame conjonctive intramusculaire

Mode

d'appréciation

Nature des caractères

Comptage Mesure $\begin{aligned} & \text { Estimation } \\ & \text { subjective }\end{aligned}$

Ensemble du Périmysium

- Importance du tissu conjonctif périmysial total

- Densité du tissu conjonctif périmysial total

- Degré de structuration

Caractères de la trame périmysiale principale

- Caractères généraux :

- Uniformité de l'épaisseur de la trame principale .....

- Epaisseur moyenne de la trame principale ...........

- Faisceaux principaux :

- Nombre de faisceaux principaux

- Forme des faisceaux principaux..... nombre de types de forme............. type de forme dominant ...............

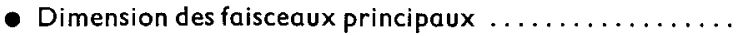

- Faisceaux principaux de petite taille ...............

- Relation forme/dimensions des faisceaux principaux .... - Paroi de la trame principale :

- Uniformité de l'épaisseur de la trame .............

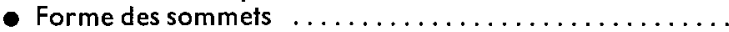

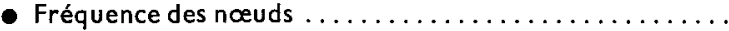

- Taille des nœuds

Caractères des trames périmysiales secondaires

- Homogénéité de la trame périmysiale secondaire ........

- Finesse des trames périmysiales secondaires ...........

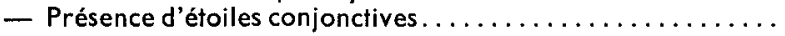

- Vaisseaux sanguins $\left\{\begin{array}{l}\text { nombre } \ldots \ldots \ldots \ldots \ldots \ldots \ldots \ldots \ldots \ldots \ldots \ldots \ldots \ldots \ldots \ldots \ldots \ldots \\ \text { taille } \ldots \ldots \ldots \ldots \ldots \ldots \ldots\end{array}\right.$

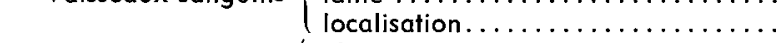

- Lames conjonctives

répartition $\ldots \ldots \ldots \ldots \ldots \ldots \ldots \ldots$

importance $\ldots \ldots \ldots \ldots \ldots \ldots \ldots$

- Taches conjonctives 
observé sont telles qu'on ne peut considérer que des parties de la trame périmysiale secondaire, au sein d'un faisceau principal (ou myoskhène).

\section{Méthodes de description des caractères définissant la structure musculaire}

Nous proposons de décrire les principaux caractères intervenant dans la définition de la structure d'un muscle au niveau de sa surface de section transversale, en considérant les différents éléments qui figurent dans la table de description mentionnée au tableau 1 et dont nous donnons ci-dessous la définition et le mode d'appréciation.

\section{TABLEAU $1 \mathrm{~B}$}

Codage des caractères estimés subjectivement dans l'ordre d'apparition dans le tableau $1 \mathrm{~A}$

Importance du tissu conjonctif périmysial

total $\ldots \ldots \ldots \ldots \ldots \ldots \ldots \ldots \ldots \ldots$

Densité du tissu conjonctif périmysial

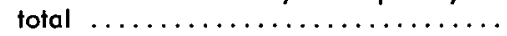

Structure atypique $\ldots \ldots \ldots \ldots \ldots \ldots$

Uniformifé de l'épaisseur de la trame ...

Epaisseur moyenne de la trame princi-

pale ......................

Types de forme parmi les faisceaux prin-

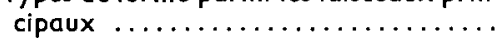
Nature de la forme dominante de ces faisceaux principaux ............

Dimension des faisceaux principaux ....

Présence de faisceaux principaux de petite taille .................. Importance des faisceaux principaux de

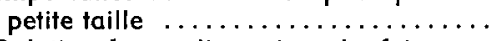
Relation forme-dimensions des faisceaux

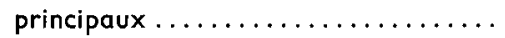

Uniformité de l'épaisseur de la trame

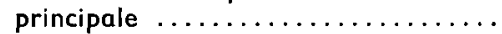
Forme des sommets . . . . . . . . .

Fréquence des nœuds ............ Taille des nœuds ................ Homogénéité de la trame périmysiale secondaire ..................

Finesse des trames périmysiales secondaires

Présence d'étoiles conjonctives ........

Taille des vaisseaux sanguins .........

Localisation des vaisseaux sanguins....

Répartition des lames conjonctives

Importance des lames par rapport au muscle........................

Taches conionctives
1 , très faible $; 2$, faible $; 3$, moyenne $; 4$, forte $; 5$, très forte.

1 , faible densité ; 2 , densité normale ; 3 , forte densité.

1 , oui ; 2 , non.

1 , répartition régulière ; 2 , répartition irrégulière.

1 , trame très fine; 2 , trame fine ; 3 , trame assez fine ; 4, trame moyenne ; 5 , trame épaisse ; 6 , trame très épaisse.

1 , forme homogène $; 2$, plusieurs types de forme.

1 , très allongée ; 2 , allongée ; 3 , compacte.

1 , très petite ; 2 , petife ; 3 , moyenne ; 4 , assez grande ; 5 , grande $; 6$, très grande.

1, pas de faisceaux ; 2 , présence de faisceaux.

1 , nulle ; 2 , faible ; 3 , moyenne ; 4 , forte.

1 , aspect homogène ef régulier ; 2 , aspect homogène mais irrégulier ; 3 , aspect hétérogène ; 4 , aspect très hétérogène.

1 , trame homogène ; 2 , trame hétérogène ; 3 , trame amincie.

1 , arrondie $; 2$, pointue.

1 , absence de nœuds ; 2 , peux noueux ; 3 , noueux $; 4$, très noueux. 1 , absence de nœuds ; 2 , petite ; 3 , moyenne ; 4 , grosse.

1 , homogène ; 2 , héłérogène.

1 , très fine ; 2 , fine ; 3 , assez fine ; 4 , moyenne ; 5 , épaisse ; 6 , très épaisse.

1 , périmysium étoilé $; 2$, périmysium non étoilé.

1 , pas de vaisseaux ; 2 , petite $; 3$, moyenne ; 4 , grosse ; 5 , très grosse.

1 , dans un coin ; 2 , au milieu ; 3 , à la périphérie ; 4 , sur un bord ; 5 , éparpillés.

1 , pas de lame ; 2 , amorce de lame sur un côté ; 3 , au milieu du muscle ; 4 , de part en part ; 5 , d̀ plusieurs endroits.

1 , pas de lame ; 2 , un quart ; 3 , un tiers ; 4 , un demi ; 5 , trois quarts ; 6, totalité.

1 , présence ; 2 , absence. 


\section{Matériel et méthodes.}

Niveau ef seuil d'observations. - Quelle que soit l'espèce animale, les muscles dont on désire analyser la structure seront examinés dans leur surface de section transversale, réalisée perpendiculairement à la direction générale de leurs fibres. Les muscles sont sectionnés minutieusement à deux niveaux distants de 20 à $30 \mathrm{~mm}$, de manière à obtenir une tranche à sections franches et parallèles. Après la coupe on procède au relevé du contour de l'échantillon placé sur une feuille de papier. Sur la section obtenue on détermine la plus grande largeur du muscle $\left(L_{1}\right)$ et l'épaisseur du muscle $\left(L_{2}\right)$ dans sa partie médiane au milieu de $L_{1}$. Ensuite la tranche est préparée

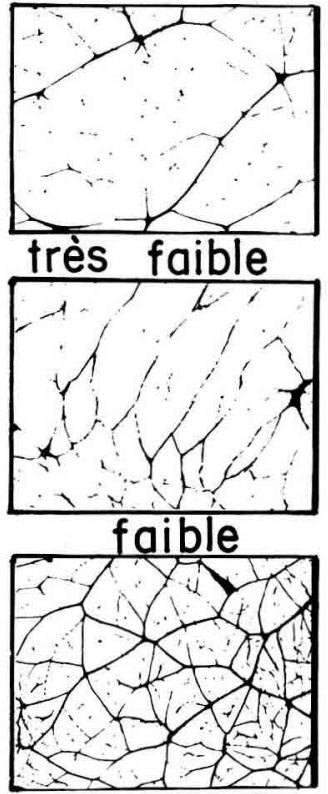

moyenne

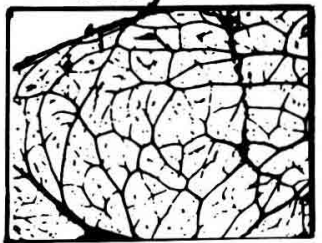

forte

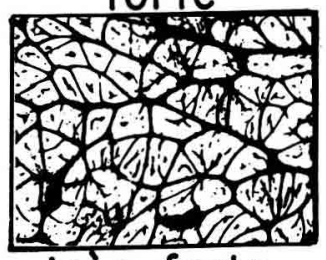

très forte

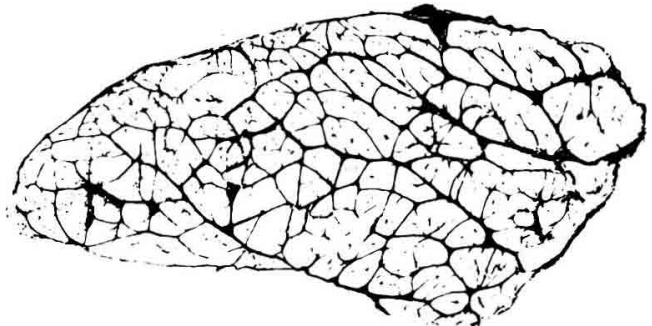

Rhomboïde
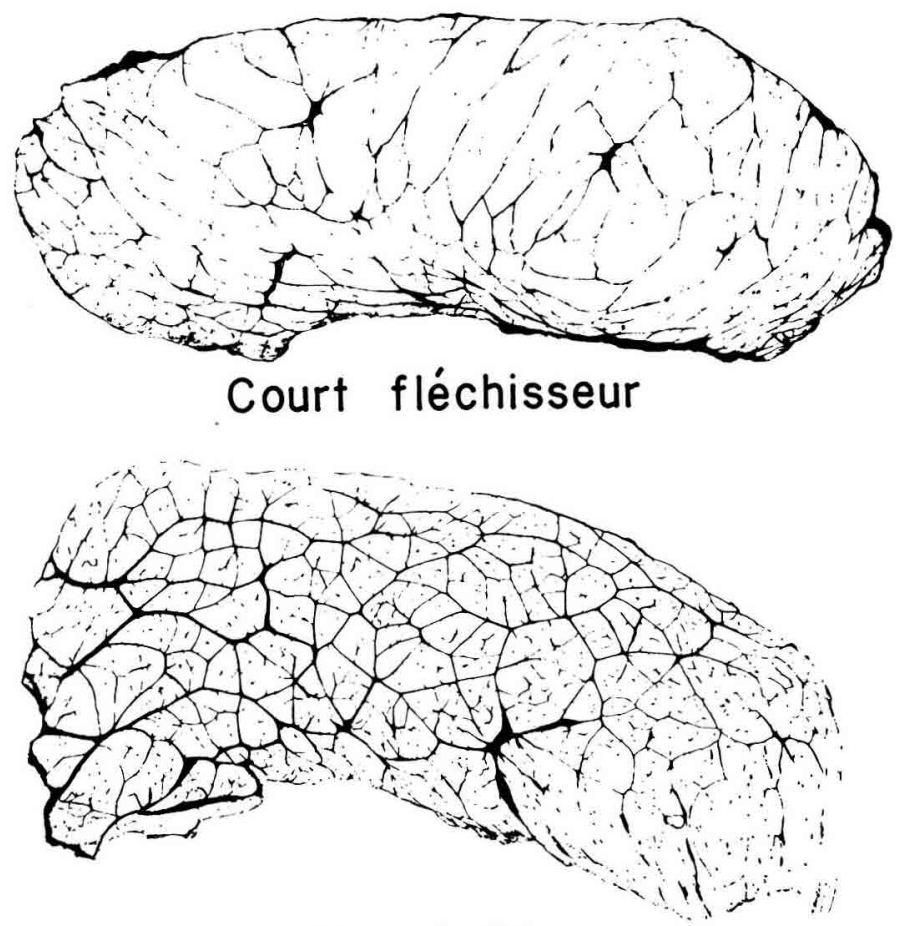

Rhomboïde

FIG. 2. - Importance du tissu conjonctif périmysial tofal. A gauche : échelle des 5 classes ;

à droite : 3 figures de muscles entiers $(\times 1)$. 
pour examens histologiques selon la méthode de Schmitt et Dumont (1969) et des coupes intéressant la totalité du muscle, de 15 micromètres d'épaisseur, sont colorées à la picro-fuchsine de Van Gieson. L'image de chaque coupe est projetée sur grand écran à l'aide d'un rétroprojecteur et l'agrandissement réalisé est de $10 \times$ linéaire. Sur l'image obtenue on procède à l'analyse descriptive de la trame conjonctive selon le cas, par comptage, mesure et appréciation subjective des différents caractères considérés. Dans ce dernier cas ceux-ci sont jugés, par comparaison aux étalons de référence décrits ci-après*.

\section{Les caractères observés.}

\section{Ensemble du périmysium.}

Importance du tissu conjonctif périmysial total.

L'importance du tissu conjonctif périmysial total peut être appréciée subjectivement et, d'une façon globale, pour l'ensemble du muscle par référence à une échelle à cinq niveaux selon la gradation suivante qualifiant l'importance estimée : 1 : très
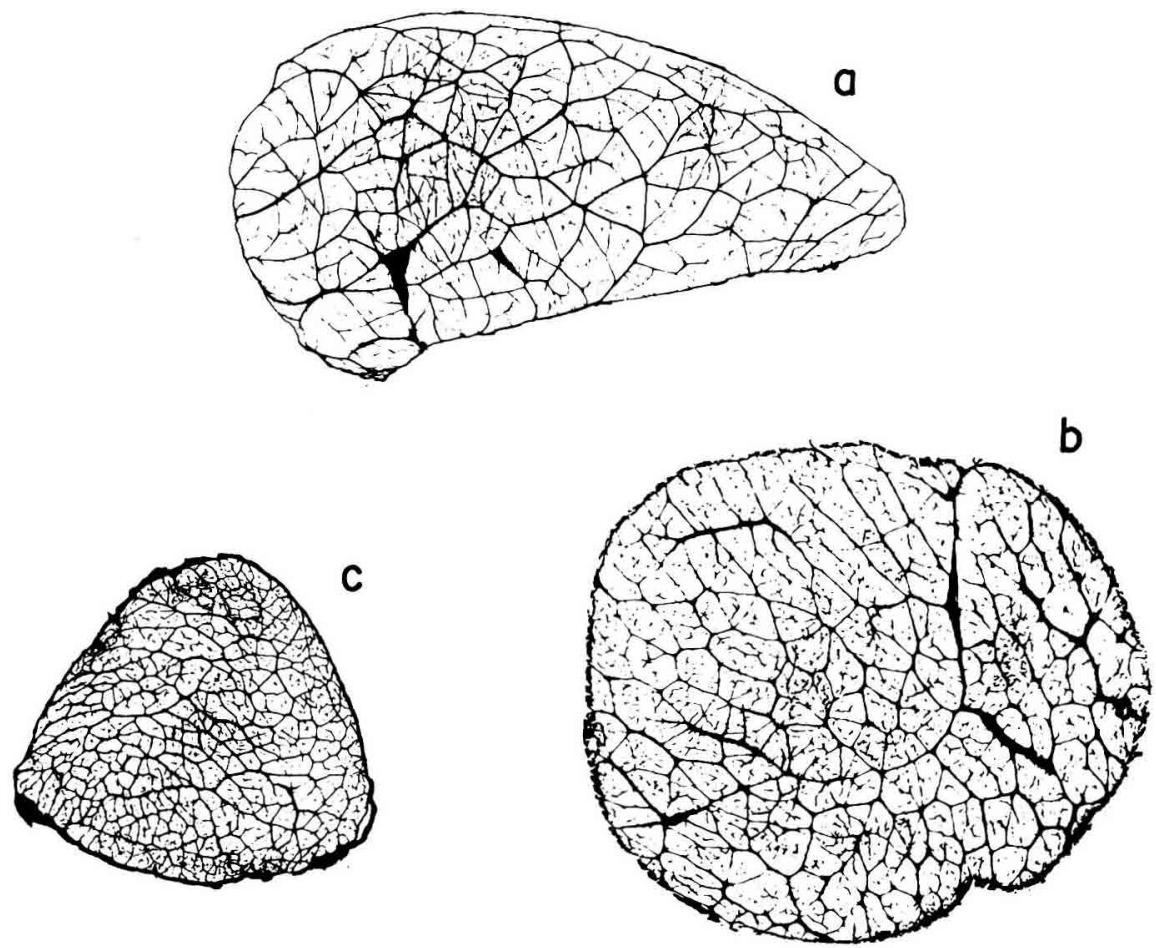

FIG. 3A. - Densité du tissu conjonctif périmysial tofal. Muscles à la taille réelle dans les trois classes. a : M. rhomboïdeus, faible densité ; $b$ : M. semitendinosus, densité normale ; c : $M$. semitendinosus, forte densité.

* A titre d'information il est précisé que les étalons de référence ont été choisis parmi les différentes éventualités offertes par l'examen de plusieurs centaines de muscles de bovins d'origines diverses. 
faible ; 2 : faible ; 3 : moyenne ; 4 : forte ; 5 : très forte. Il n'est pas possible, matériellement, de représenter ici au grandissement souhaité ( $\times 10$ linéaire) les étalons de référence établis au Laboratoire et considérés comme représentatifs de chacune des cinq classes de l'échelle. On aura cependant une idée de leur valeur en considérant les illustrations de la figure 2 qui présente en grandeur nature chacune de ces cinq classes.

a
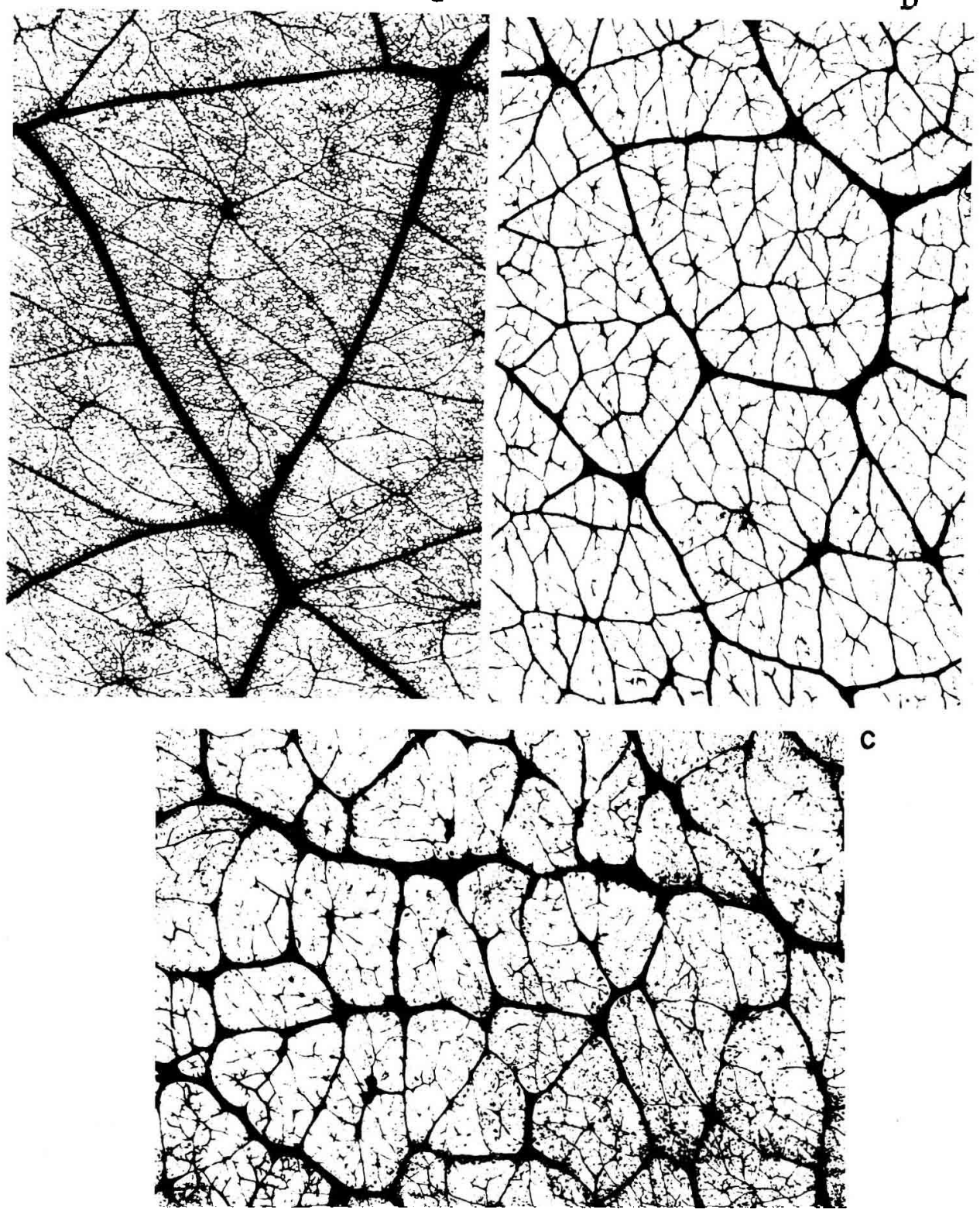

FIG. 3B. - Densité du tissu conjonctif périmysial tołal : mêmes muscles que figure 3A, grossis 10 fois (linéaire). a : faible; b : normale ; $c$ : dense. 
Densité du tissu conjonctif périmysial total.

Le jugement de l'importance globale du périmysium précédemment envisagé s'applique à une estimation de l'ensemble du conjonctif périmysial, considéré de façon absolve. 11 peut paraître intéressant de préciser ce jugement en considérant la « densité » du périmysium, c'est-à-dire en appréciant quelle est l'importance du périmysium par unité de surface du muscle. Sur cette base on peut retenir au moins les trois éventualités suivantes : 1 : faible densité ; 2 : densité normale ; 3 : forte densité. La densité sera d'autant plus marquée que le nombre de faisceaux principaux est plus élevé et que la division des faisceaux principaux en faisceaux d'ordres inférieurs est plus prononcée. La figure 3 donne des exemples des trois cas de densité.

\section{Structuration du tissu conjonctif périmysial.}

- Notion de structuration. - La figure $4 a$ représente une portion de section d'un muscle observé à un grandissement linéaire de 10. Les fibres musculaires sont groupés en faisceaux dits faisceaux primaires, que nous appellerons ici faisceaux d'ordre 1. Chacun des faisceaux est entouré par un périmysium de faible importance. C'est l'emplacement de ces différents faisceaux qui est présenté dans la figure $4 b$ où l'on a dessiné avec un trait d'épaisseur uniforme le contour des faisceaux primaires. Un nombre variable de faisceaux d'ordre 1 peuvent être regroupés pour constituer un faisceau de beaucoup plus grande taille, faisceau secondaire ou faisceau d'ordre 2 délimité par un périmysium nettement plus marqué que celui des faisceaux d'ordre 1. La figure 4c représente les faisceaux d'ordre 2 qui ont été identifiés et dont on a dessiné le contour avec un trait d'épaisseur plus marquée. On peut considérer qu'à leur tour un certain nombre de faisceaux d'ordre 2 sont rassemblés pour donner un faisceau tertiaire ou faisceau d'ordre 3, au périmysium encore plus important. La figure $4 d$ représente les faisceaux d'ordre 3 qui apparaissent à l'observateur et dont on a dessiné le contour avec un trait d'épaisseur uniforme et assez important. Enfin les six faisceaux d'ordre 3 peuvent être regroupés en deux faisceaux d'ordre 4, séparés par une importante travée de périmysium représentée sur la figure $4 \mathrm{e}$ par un traif épais. L'ensemble des deux faisceaux d'ordre 4 constitue (fig. $4 f$ ) un faisceau principal (ou unité d'organisation ou myoskhène). D'une façon générale un ensemble de faisceaux d'ordre $n-1$ constitue un myoskhène d'ordre $n$. La valeur de l'ordre des faisceaux principaux traduit ce que nous appelons le degré de structuration du muscle. Dans le cas de la figure $4 a$ ce degré de structuration a la valeur 5 .

- Appréciation du degré de structuration. - Le degré de structuration d'un muscle peut être calculé à partir de la moyenne des numéros d'ordre des différents faisceaux principaux du muscle. Un mode plus rapide, mais moins précis, d'estimation de la structuration a déjà été proposé (Schmitt et Dumont, 1969). Le nombre des différentes classes d'épaisseur de périmysium présentes autour et au sein d'un faisceau principal représente en effet l'ordre du myoskhène. En procédant à sa détermination sur une dizaine de faisceaux différents et en retenant la moyenne, on obtient une valeur suffisamment précise du degré de structuration du muscle.

- Structuration atypique. - Dans la plupart des cas il est facile de distinguer les différents ordres de faisceaux, d'après l'épaisseur des parois du périmysium ef l'on observe normalement une progression régulière de cette épaisseur en passant des parois interfasciculaires d'ordre 1 aux parois interfasciculaires d'ordre 2, puis d'ordre 
3 etc... pour arriver finalement à l'épaisseur des parois interfasciculaires d'ordre $n$ qui séparent les divers myoskhènes et qui correspondent à la trame périmysiale principale.

a
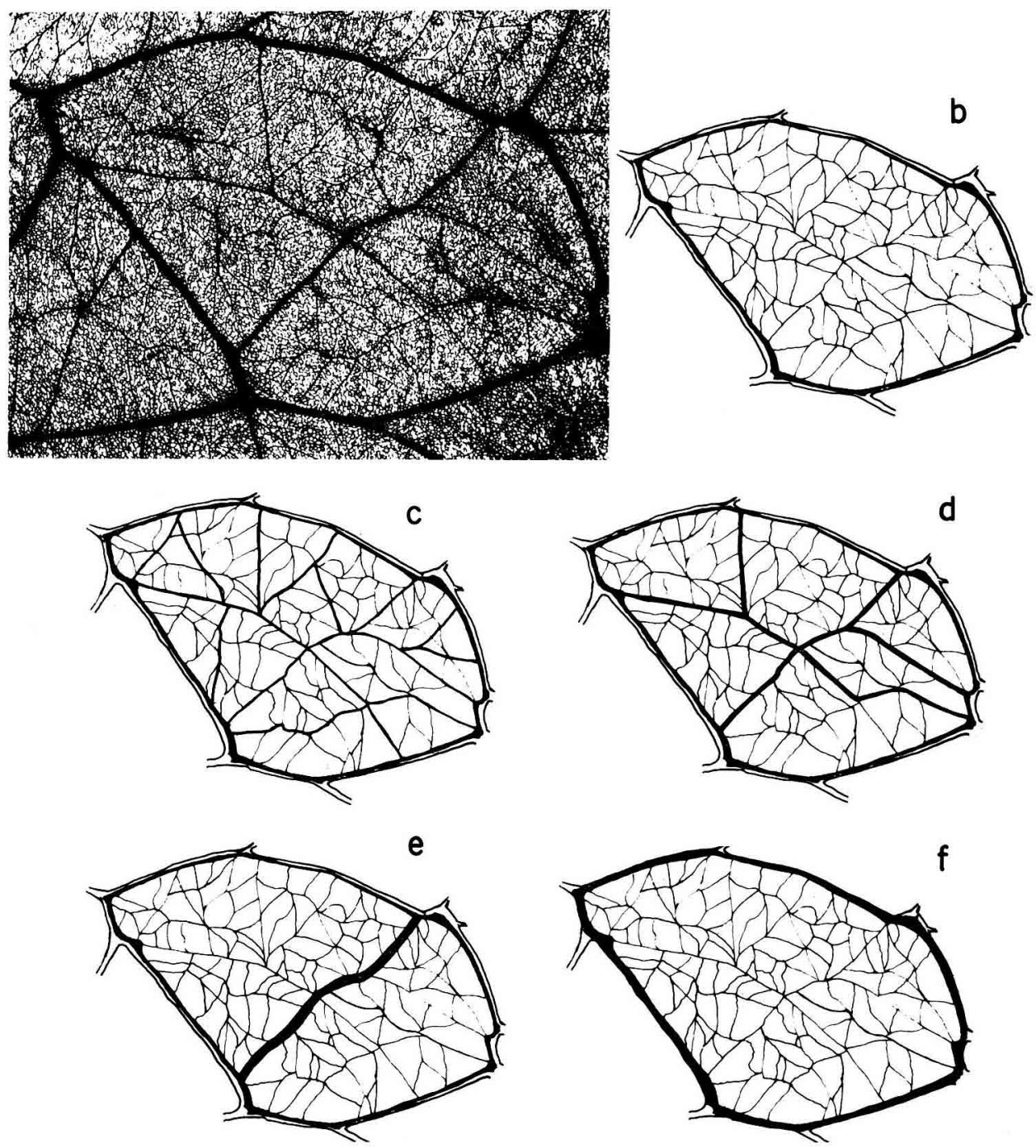

FIG. 4. - Notion de structuration du tissu conjonctif périmysial.

Dans certains muscles les parois interfasciculaires d'ordre n-1 paraissent manquer, de sorte que l'on passe, sans transition dans les épaisseurs, des parois interfasciculaires encore assez fines des faisceaux d'ordre $n-2$ à la paroi relativement plus épaisse du faisceau principal. On peut parler alors de structuration atypique, par opposition à la structuration normale où la hiérarchie des différents types d'épaisseurs est 
bien respectée. Le qualificatif d'atypique retenu pour décrire cefte situation tient compte du fait qu'il s'agit là d'une condition beaucoup moins fréquente. Dans le cas d'une structuration atypique, la trame périmysiale principale se distingue très facilement et l'intérieur des faisceaux principaux se caractérise par la finesse de son périmysium (fig. 5).

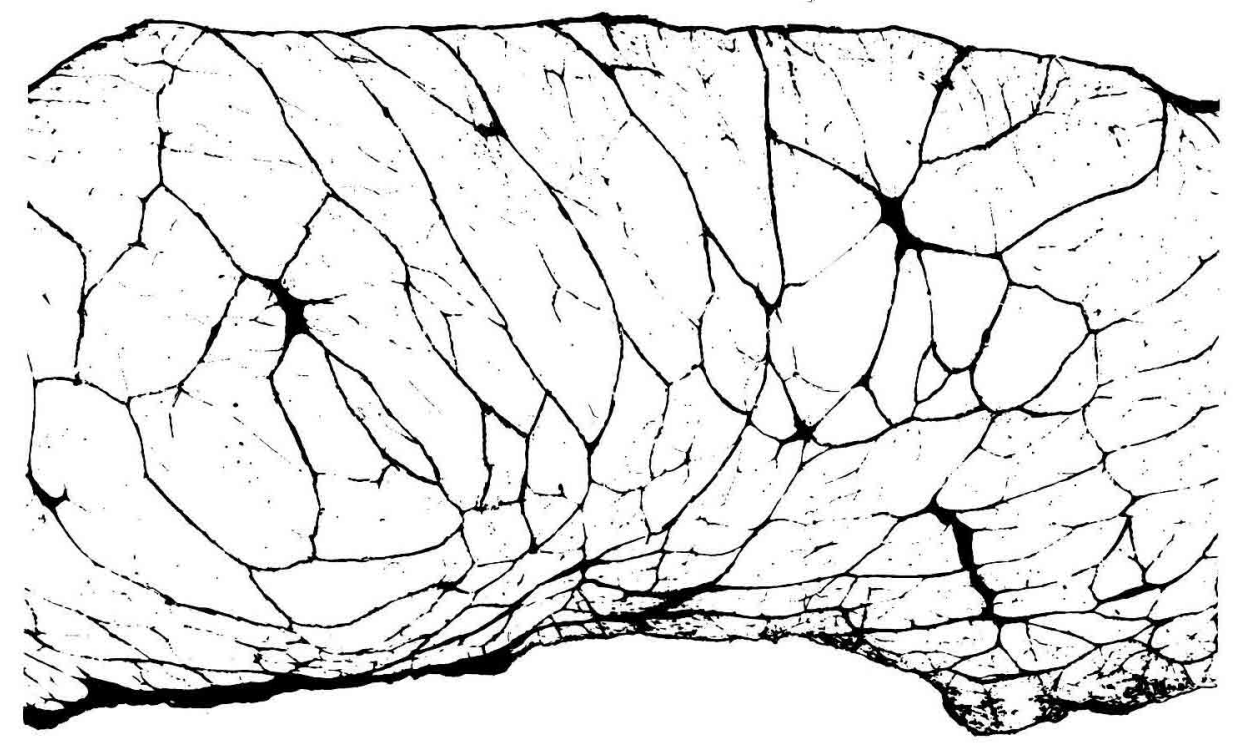

FIG. 5. - Exemple de muscle possédant une structure atypique.

Caractères de la trame périmysiale principale.

Caractères généraux.

- Uniformité de son épaisseur. Notion de « régularité » de la trame. - Ainsi qu'il a été rappelé précédemment, la trame principale du périmysium se distingue par son épaisseur relativement uniforme et par la régularité de sa répartition. L'épaisseur de cette trame est généralement plus importante que celle des faisceaux d'ordre inférieur (primaire, secondaire...). Elle est souvent aussi assez uniforme d'une région à l'autre du muscle. Dans ce cas on parle de répartition régulière de la trame. A l'opposé on peut trouver, dans certains muscles, des trames principales présentant d'une région à l'autre des épaisseurs différentes. On est alors en présence d'une répartition irrégulière (fig. 6).

- Epaisseur moyenne de la trame périmysiale principale. - On considère comme épaisseur «moyenne » de la trame, et en quelque sorte comme épaisseur représentative, celle qui intéresse la majeure partie de la surface de section, dans le cas d'une trame " irrégulière " ou la totalité de la section dans le cas d'une trame « régulière ». Selon les muscles, cette épaisseur moyenne est très variable ef l'on rencontre tous les intermédiaires possibles entre une trame très fine et une trame très épaisse. Lorsque la trame est très fine sa caractérisation à l'œil nu est difficile ef sa reproduction sur 
document (par exemple photographies ou épreuves d'imprimerie) devient aléatoire. C'est le cas pour le muscle a de la figure 7. A l'opposé, lorsque la trame est relativement épaisse, elle se distingue facilement en donnant au muscle une structure grossière (cf. muscle f de la figure 7). Il est proposé d'apprécier subjectivement l'épaisseur moyenne de la trame périmysiale principale par référence à l'échelle indiquée au tableau 1B qui prévoit six évenfualités, considérant la trame comme très fine, fine, assez fine, moyenne, épaisse ou très épaisse. Ces qualificatifs s'appliquent à des trames dont les épaisseurs seront les suivantes :

\section{Qualificatif Epaisseur moyenne de la trame}

$$
\text { (en } \mu \mathrm{m})
$$

trame très fine

trame fine

trame assez fine

trame moyenne

trame épaisse

trame très épaisse
$<50$

50 à 100

100 d̀ 150

150 à 250

250 d̀ 400

$>400$
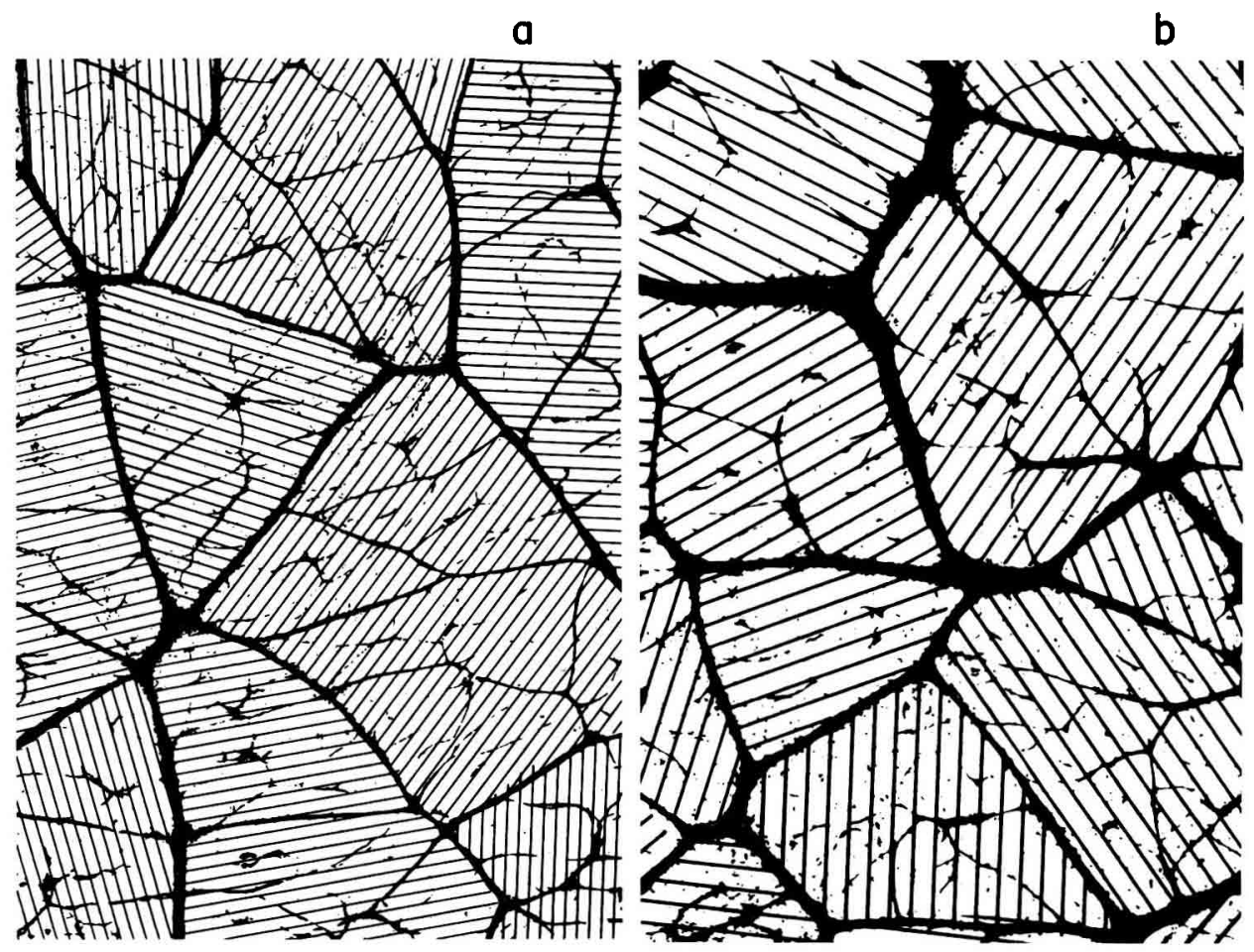

FIG. 6. - Répartition de l'uniformité de la trame périmysiale principale. a : répartifion régulière, b : répartition irrégulière (les faisceaux principaux ont été hachurés afin de les différencier). 

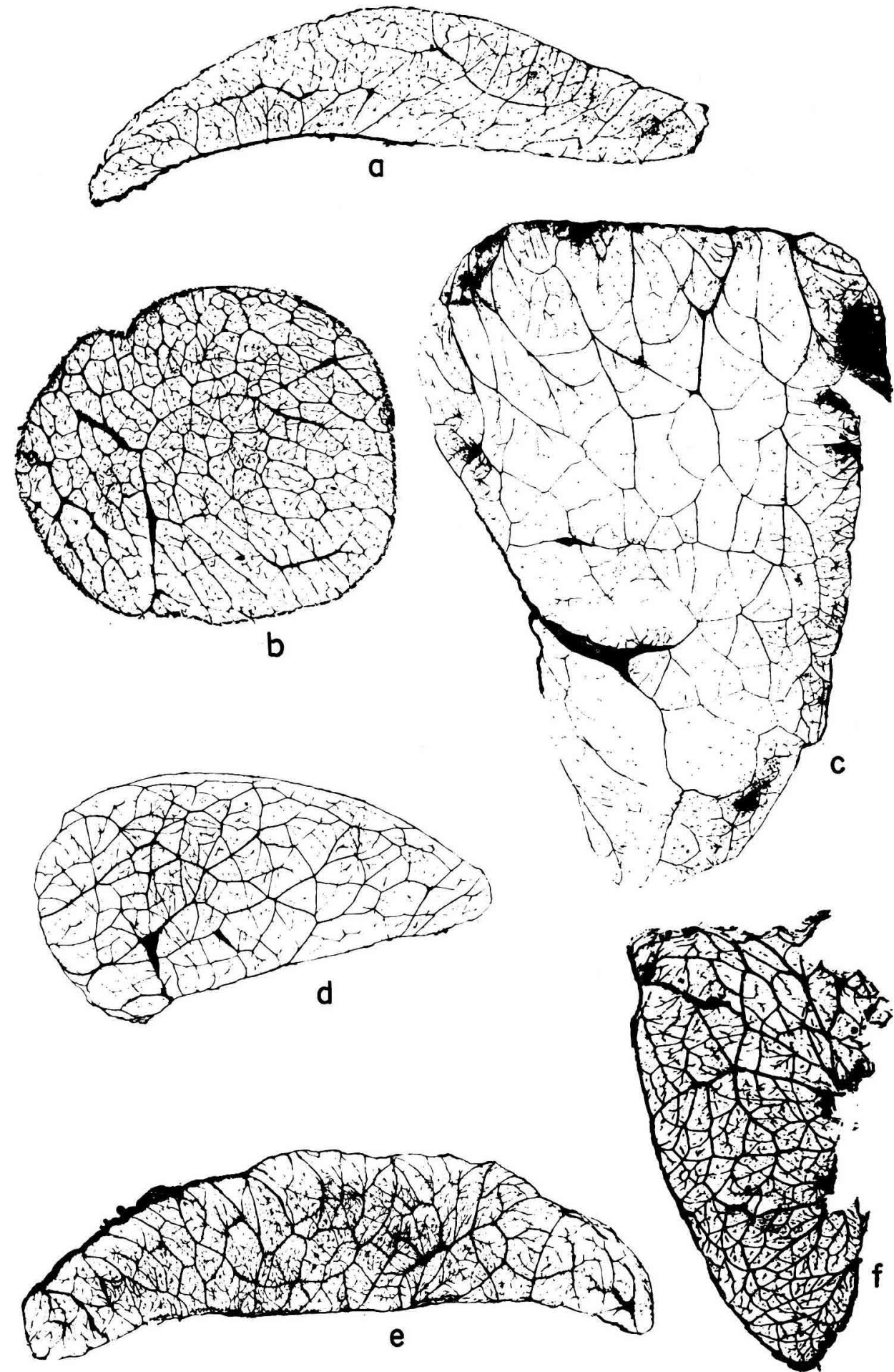

FIG. 7. - Epaisseur moyenne de la trame périmysiale principale dans les six classes de l'échelle de jugement $(\times 1)$. 
Les faisceaux principaux.

- Nombre de faisceaux principaux. - Le nombre des faisceaux principaux peut être déterminé exactement par comptage. L'opération est assez facile et rapide à effectuer lorsqu'on procède sur documents photographiques, où le repérage de la trame a été soigneusement exécuté au préalable et éventuellement souligné, en trait renforcé, sur le document de travail. On a ainsi précisé le nombre de faisceaux principaux de muscles de porcins de différentes races (Dumont et Schmitt, 1970) ou de bovins de différents âges.

Pour une évaluation plus rapide du nombre des faisceaux dans les essais comparatifs systématiques, on peut ne compter que le nombre de faisceaux principaux distribués dans une direction particulière ou localisés dans une portion donnée du muscle. II est ainsi envisagé de considérer le nombre de faisceaux principaux présents le long des deux axes principaux de la surface de section $\left(L_{1}\right.$ et $\left.L_{2}\right)$ précédemment définis et déterminés sur le muscle. La figure 8 présente un exemple de dénombrement des faisceaux principaux $M_{1}$ selon $L_{1}$ et $M_{2}$ selon $L_{2}$ dans le cas du muscle $M$. semitendinosus de bovin.

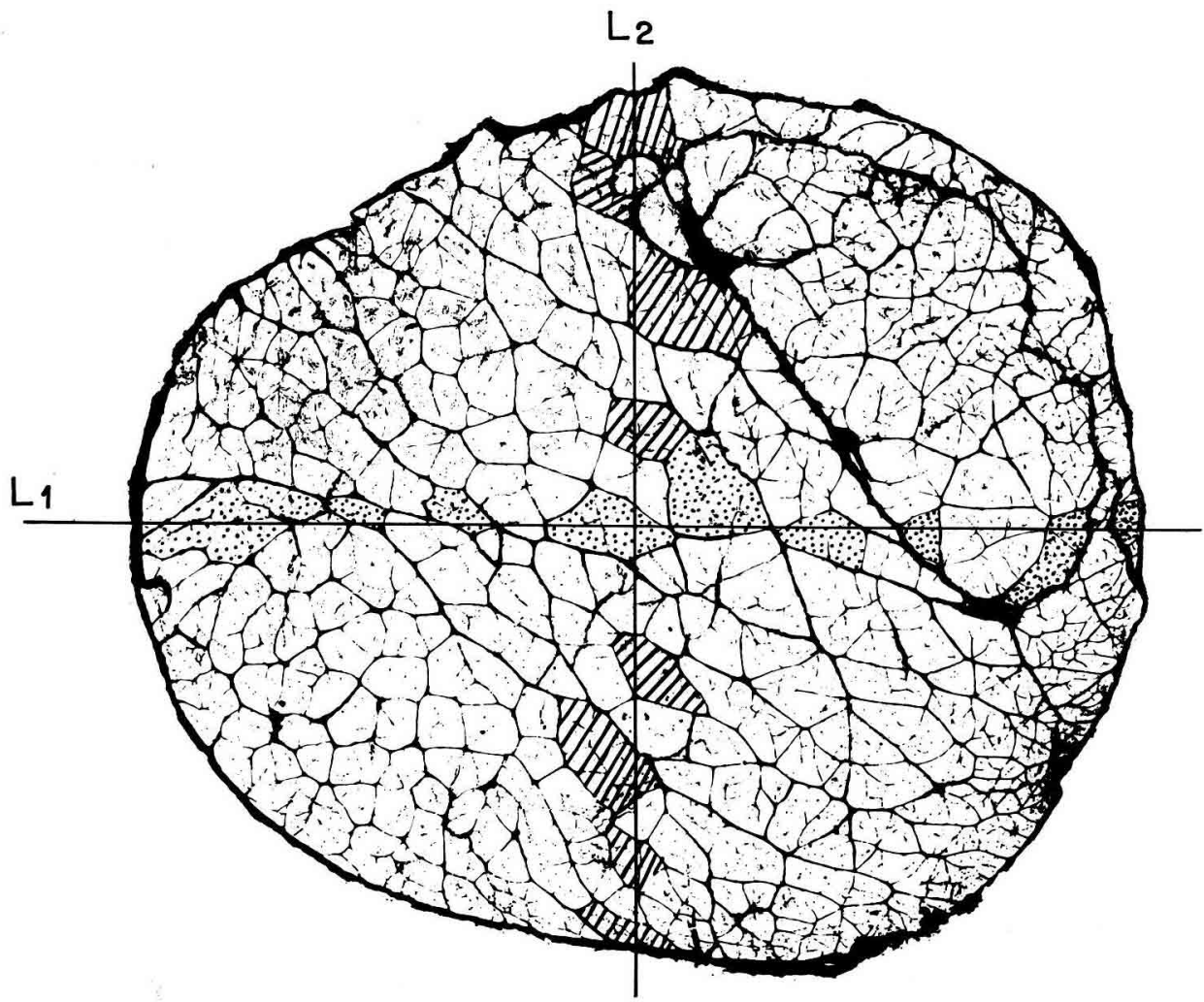

G. 8. - Dénombrement de faisceaux principaux selon deux axes. L'axe horizontal nous donne 17 unités (une sur deux en pointillé). L'axe vertical dénombre 18 unités (une sur deux hachurée) $(\times 1)$. 
- Forme des faisceaux principaux. - La forme des faisceaux principaux est assez variable, à l'intérieur d'un muscle ef d'un type de muscle à l'autre. Les formes rencontrées sont celles d'éléments polygonaux simples, du triangle à l'hexagone. Les différentes figures qui illustrent le texte donnent des exemples des formes rencontrées (cf. fig. 1). Il semble en fait que, sous le couvert de cette variabilité, on puisse distinguer 3 catégories auxquelles les formes des faisceaux principaux sont assimilables, selon le rapport de leurs dimensions extrêmes :

- forme compacte : lorsque le rapport de la plus grande dimension à la plus petite est de l'ordre de 1 à 1,5; dans ce cas l'impression générale laissée par l'examen de l'ensemble des faisceaux est que ceux-ci sont composés de différents types de polygones réguliers;

- forme allongée : lorsque le rapport de la plus grande dimension à la plus petite est de l'ordre de 1,5 à 2 ;

- forme très allongée : lorsque le rapport de la plus grande dimension à la plus petite est supérieure d̀ 2 .

Ełant donné la variabilité des formes rencontrées dans l'ensemble d'un muscle, il est délicat, voire impossible, de parler d'une forme « moyenne » des faisceaux principaux. Pour traduire, cependant, l'allure générale de la forme des faisceaux principaux on peut retenir :

- soit de caractériser le type de forme (compacte, allongée ou très allongée) après mesures des deux dimensions de chaque faisceau principal ef d'indiquer la fréquence absolue ou relative de chacun des types;

- soit, plus rapidement, de juger subjectivement l'ensemble ef de retenir comme

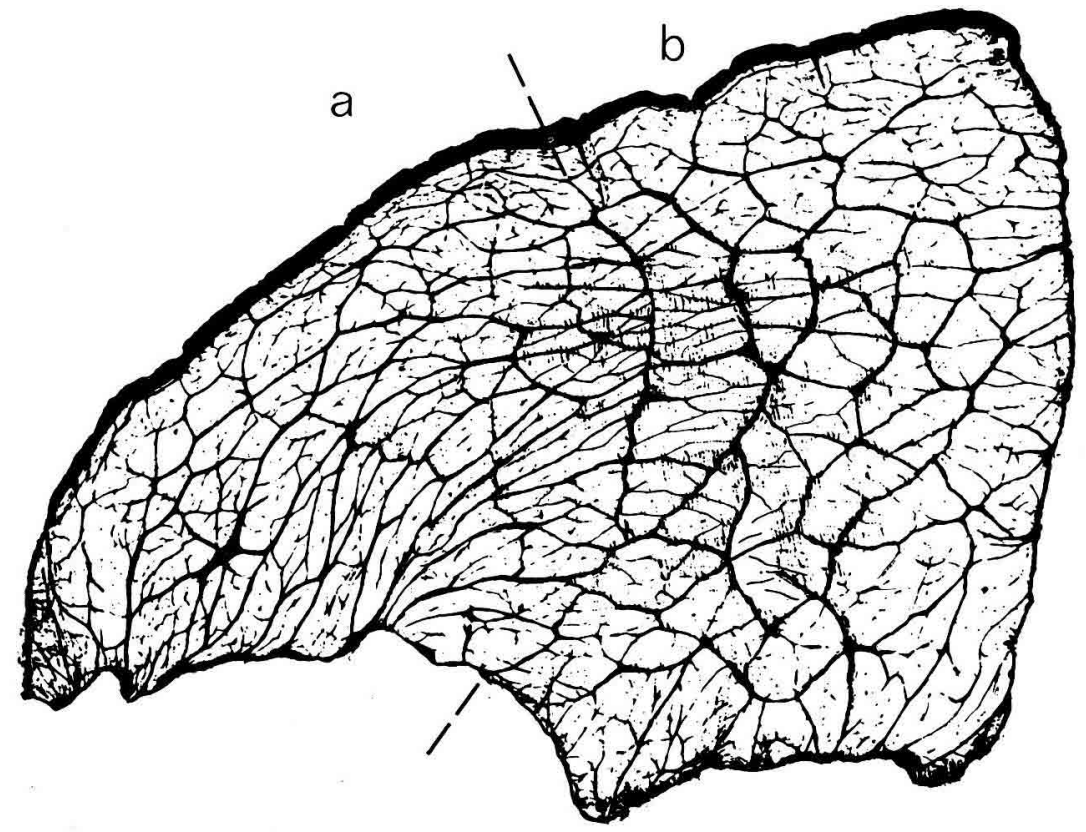

FIG. 9. - Muscle ayant deux types de forme. $a$ : très allongée ; $b$ : compacte. 
type de forme le type dominant. En règle générale, parmi les différents types de forme observés dans un muscle, l'expérience montre que l'un d'eux apparaît comme le plus abondant et que sa fréquence dépasse nettement 50 p. 100. On peut alors le considérer comme le type de forme dominant.

Dans certains cas on peut estimer que la totalité ou la quasi-totalité des faisceaux ont - indépendamment de leurs dimensions absolves - le même type de forme, d'un faisceau à l'autre (fig. $7 b$ ). On pourra alors traduire de façon particulière cette homogénéité en indiquant, selon le code mentionné au tableau $1 \mathrm{~B}$, qu'on ne rencontre qu'un type de forme de faisceau dans le muscle. On opérera de même en cas d'hétérogénité (fig. 9).

- Dimensions des faisceaux principaux. - La trame principale du périmysium définit un réseau dont la grandeur des mailles est également très variable, spécialement d'un muscle à l'autre.

On peut déterminer précisément la grandeur des mailles par mesure de la plus grande dimension des faisceaux principaux (et mesure de la plus petite dimension) comme on peut le faire pour les fibres musculaires à titre individuel ; on peut aussi en mesurer exactement la surface.

L'estimation rapide de la dimension «moyenne » des faisceaux au niveau de l'ensemble de la coupe peut être envisagée par un jugement subjectif global, par référence à une échelle de variation, comme celle qui est proposée dans la figure 10. L'échelle comprend six classes, correspondant aux définitions suivantes :

1 : faisceaux principaux très petits ;

2 : faisceaux principaux petits ;

3 : faisceaux principaux moyens ;

4 : faisceaux principaux assez grands ;

5 : faisceaux principaux grands;

6 : faisceaux principaux très grands.

La correspondance avec la taille réelle des faisceaux principaux est la suivante :

\begin{tabular}{|c|c|}
\hline Longueur maximum (en $\mathrm{mm}$ ) & Classe \\
\hline$\leqslant$ & 1, très petits \\
\hline 3,1 à 6 & 2, petits \\
\hline 6,1 à 9 & 3 , moyens \\
\hline 9,1 à 12 & 4, assez grands \\
\hline 12,1 à 15 & 5 , grands \\
\hline$>15$ & 6. très grands \\
\hline
\end{tabular}

Compte tenu de la variabilité enregistrée dans la dimension des faisceaux, il est difficile, voire impossible, d'en définir une valeur moyenne qui ait, en dehors de son sens mathématique, une signification réelle au plan de la structure. A cel égard on peut souligner l'intérêt que présente le jugement subjectif global pour l'estimation de la classe de dimension dominante, en tenant compte, là aussi (d'après l'expérience acquise), qu'en règle générale les faisceaux principaux appartiennent, au moins pour 70 à 80 p. 100 d'entre eux, à la même classe de dimension. 


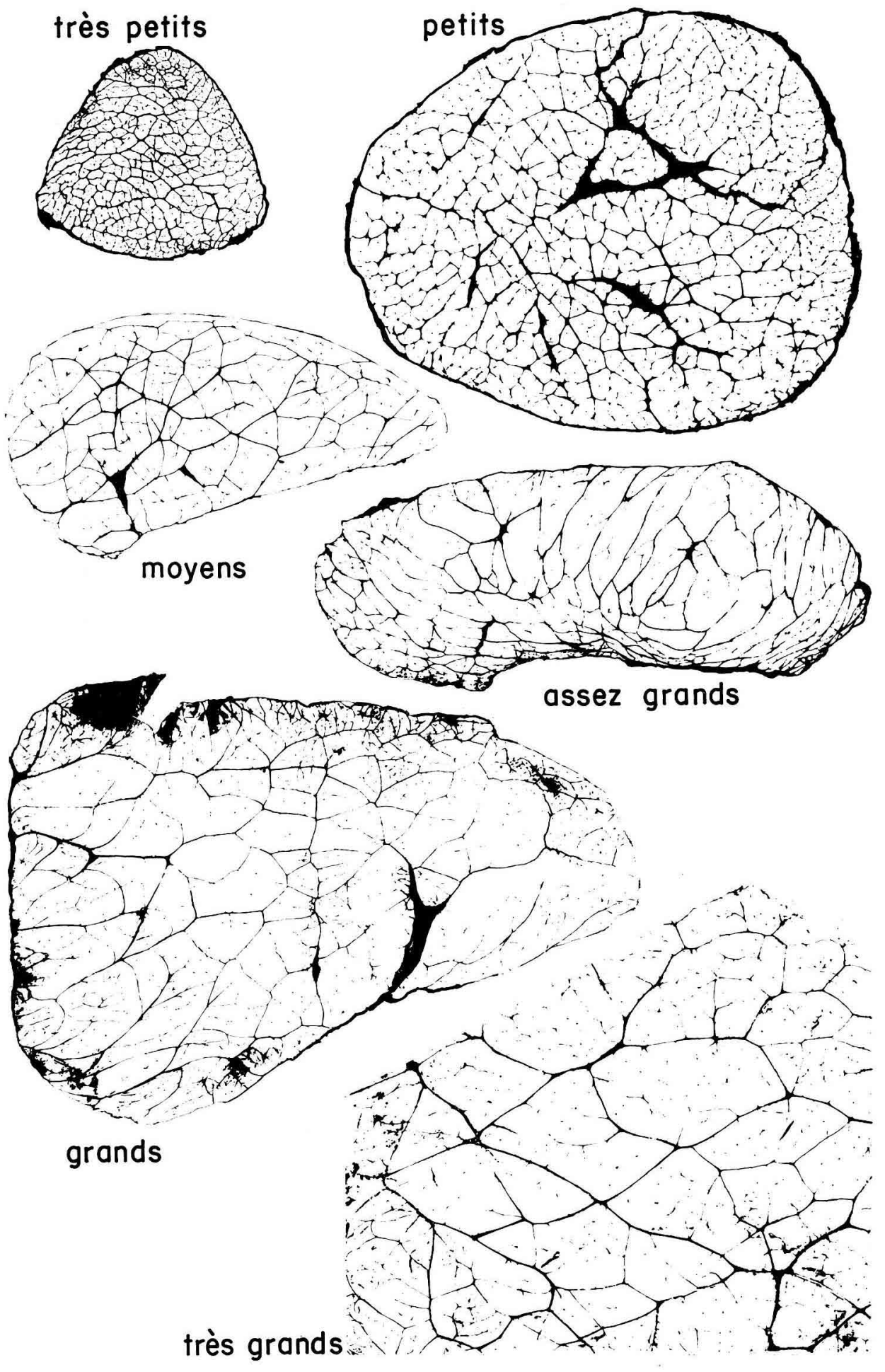

FIG. 10. - Dimension des faisceaux principaux $(\times 1)$. 
Une estimation indirecte de la dimension des faisceaux principaux peut être obtenue aussi en divisant la largeur $L_{1}$ ou l'épaisseur $L_{2}$ du muscle par le nombre de faisceaux principaux traversés $\left(M_{1}\right.$ ou $\left.M_{2}\right)$. Le rapport $L_{1} / M_{1}$ est une valeur indicatrice de la taille des faisceaux principaux selon la largeur ef le rapport $L_{2} / M_{2}$ selon l'épaisseur.

- Faisceaux principaux de pefite taille. - Une des causes de l'irrégularité de l'aspect de la trame principale du tissu conjonctif est la présence, à côté des faisceaux principaux de taille moyenne ou grande (correspondant aux classes 3 à 6 décrites supra), de faisceaux principaux de petite dimension (classes de taille 1 à 2 ). Quand ils existent, ces faisceaux se distinguent aisément. Leur localisation, selon les muscles, paraît variable. Dans un premier temps il convient de s'assurer de leur présence (et de coder l'éventualité correspondante comme indiquée dans le tableau 1B). Leur répartition peut être décrite en clair de façon détaillée par rapport aux dimensions du muscle. La figure 11 donne l'exemple d'un muscle présentant des faisceaux principaux de petite taille, localisés sur l'un des bords du muscle.

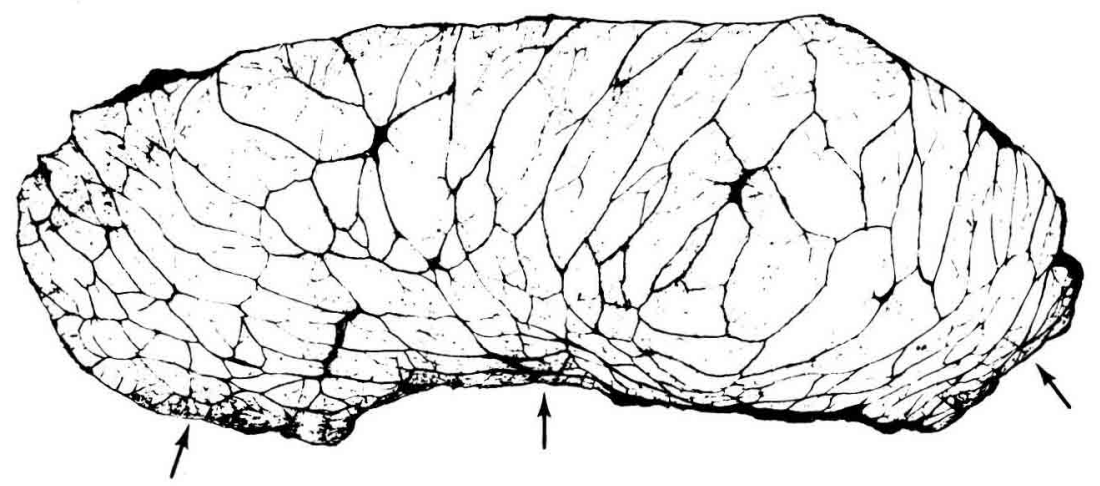

FIG. 11. - Faisceaux principaux de petife faille (localisation : $\uparrow)(\times 1,5$ linéaire).

- Relations forme/dimensions des faisceaux principaux. - La variabilité de la structure du muscle que l'on rencontre en comparant différents échantillons s'explique, pour une large part, par les différences dans la forme des faisceaux principaux et dans leurs dimensions. L'identité de formes des faisceaux (quelle qu'en soit la taille)

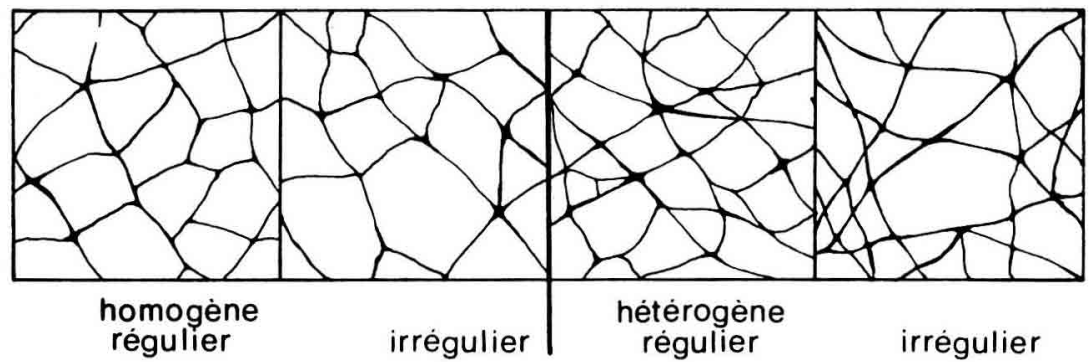

FIG. 12. - Répartition des types de forme des myoskhènes $(\times 1)$. 
confère à l'aspect de la section une grande homogénéité. A cet égard l'expérience montre qu'on peut effectivement rencontrer les différentes éventualités, théoriquement envisageables à partir de la variation de la forme et de la variation dans les dimensions, ef qui se ramènent à quatre cas de figures illustrés dans la figure 12.

1) L'aspect général des figures géométriques des unifés de structure est homogène et uniforme; dans leur ensemble, les unités ont des dimensions et des formes voisines. On peut parler d'un aspect homogène et régulier d'une trame isomorphe et isométrique.

2) L'aspect général des figures est toujours homogène en ce qui concerne la forme mais la trame esł composée d'unités de tailles différentes : on parle d'un aspect homogène et irrégulier d'une trame isomorphe ef hétérométrique.

3) L'aspect général est hétérogène ; les faisceaux sont de deux types de formes et de dimensions différentes ; toutefois ces diverses unités sont regroupées, assez régulièrement, en ensembles délimitant, au sein du muscle, des motifs assez semblables (comme ceux que l'on trouve sur du papier peint). On peut parler d'une trame « hétérogène mais régulière $»$.

4) L'aspect général est très hétérogène en raison d'une grande variabilité dans la forme et la taille des différents faisceaux : la trame est hétérogène ef d'allure irrégulière.

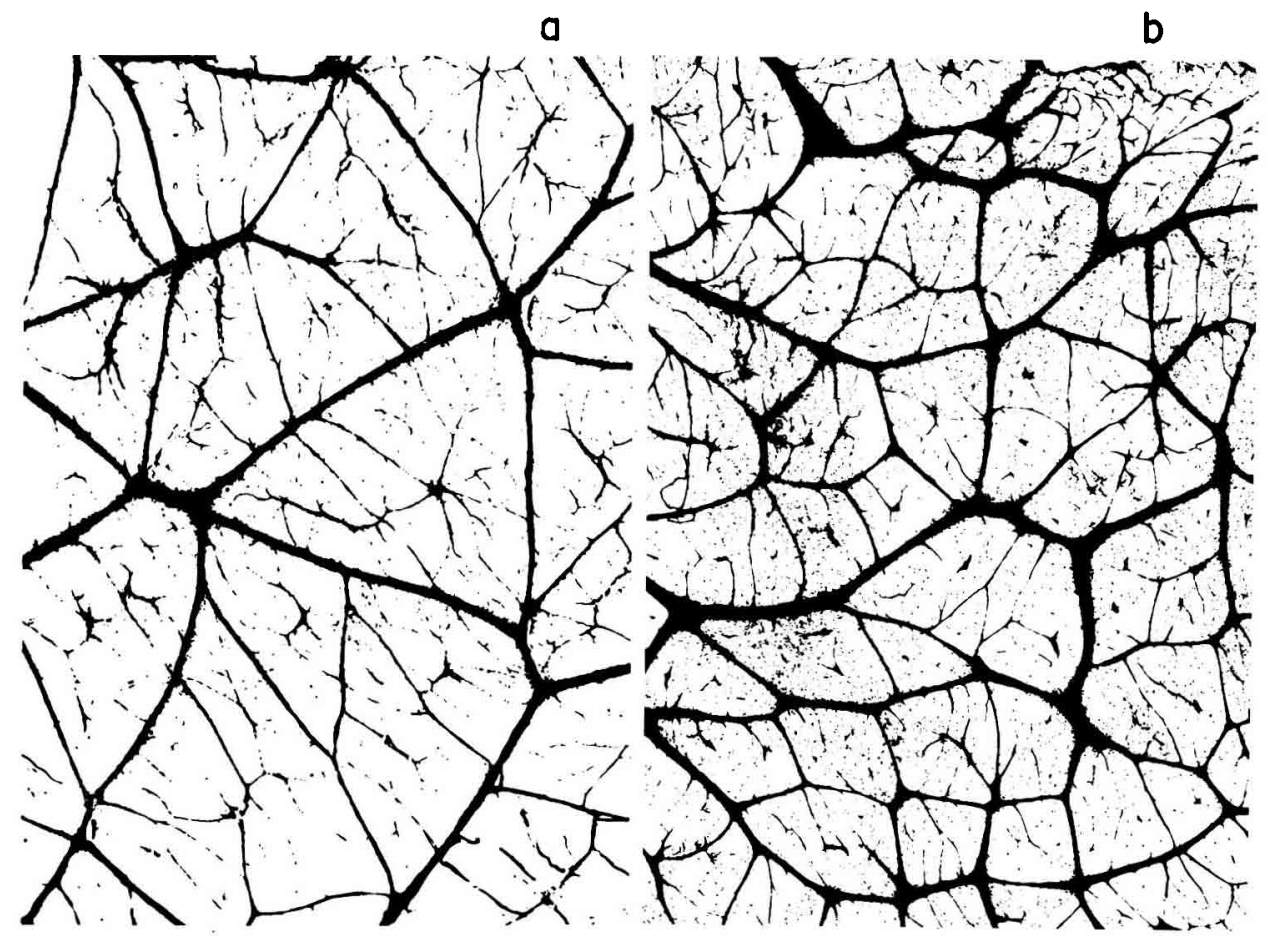

FIG. 13. - Uniformifé de l'épaisseur de la frame principale. $a$ : uniforme ; $b$ : non uniforme. 
Caractères des parois de la trame principale du périmysium.

- Uniformité dans son épaisseur. - La trame principale du périmysium peut être dans une zone donnée de la surface de section, soit très homogène et uniforme dans son épaisseur, soit au contraire hétérogène pour ce caractère. Dans ce dernier cas (fig. 13b) les parois de la trame sont d'épaisseur très variable d'une portion à l'autre de la trame. Lorsque la trame est uniforme (fig. 13a) les parois se présentent de façon régulière, les deux bords de chaque paroi étant parallèles. Les figures (13a et $13 b)$ illustrent ainsi ces deux types d'éventualités, qu'on peut retenir dans la table de description (tabl. 1B).

On observe dans certains muscles une modification particulière des contours de la trame périmysiale principale, telle que dans la partie centrale des parois, l'épaisseur de la trame soit régulière ef uniforme mais très réduite. Par contre, au niveau des sommets des faisceaux principaux, l'épaisseur de la trame est renforcée, au point de constituer des nœuds d'assez forte taille (fig. 14). L'amincissement des parois des faisceaux principaux est un caractère intéressant à relever dans la table de description.

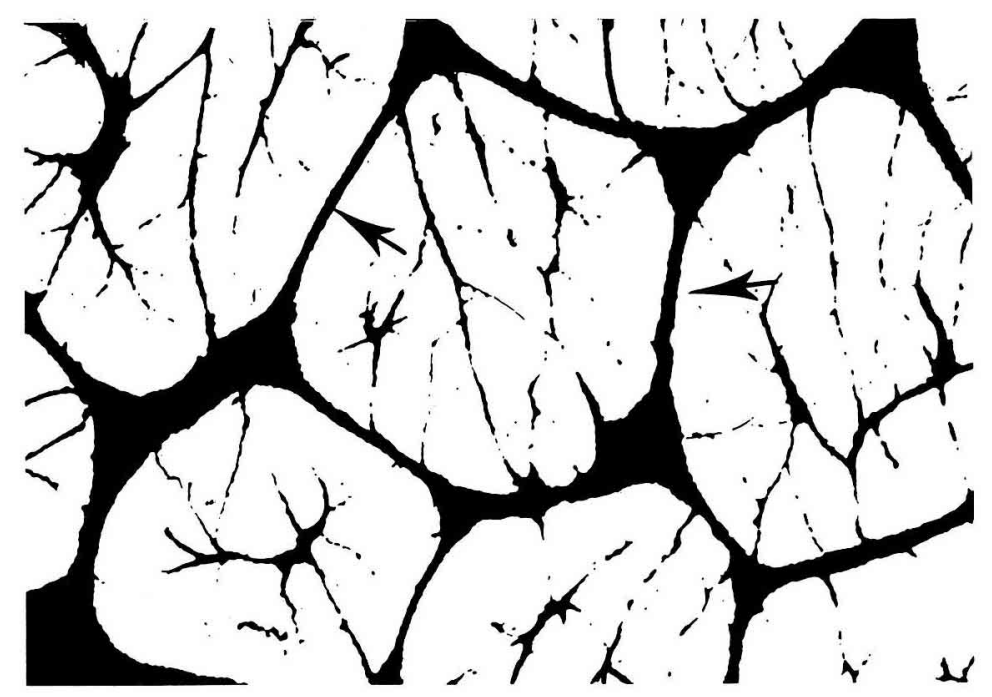

FIG. 14. - Exemple de trame amincie ( $\times 10$ linéaire).

- Forme des sommets. - La forme des parois de la trame conjonctive détermine la forme des sommets des divers types de polygones dessinés par la trame et qui correspondent aux myoskhènes ou faisceaux principaux. Selon les cas, on peut rencontrer soit des sommets dont les arêtes sont plus ou moins vives (forme pointue ou angle franc), soit des sommets plus ou moins arrondis. Ces deux types de forme des sommets des faisceaux principaux sont représentés dans la figure 15 .

- Les nœuds. - Au niveau des jonctions des différents faisceaux principaux la trame principale peut présenter des renflements et des épaississements plus ou moins importants et que nous proposons d'appeler des « nœuds». On peut caractériser ces 
nœuds à la fois par leur fréquence relative, sur l'ensemble de la section et par leur taille. Il est proposé de procéder à une estimation subjective de ces deux caractères au niveau de l'ensemble de la surface du muscle, selon l'échelle mentionnée au tableau 1B qui envisage quatre éventualités pour la fréquence (pas de nœuds, peu noueux, noveux, très noveux) et trois pour la faille (nœuds petits, moyens ou gros). Dans ce cas on peut retenir que les petits nœuds sont ceux qui ont moins de $0,3 \mathrm{~mm}$ de large et les gros ont plus de $0,8 \mathrm{~mm}$ de large. La figure 16 présente trois types d'images rencontrées en ce qui concerne les nœuds.
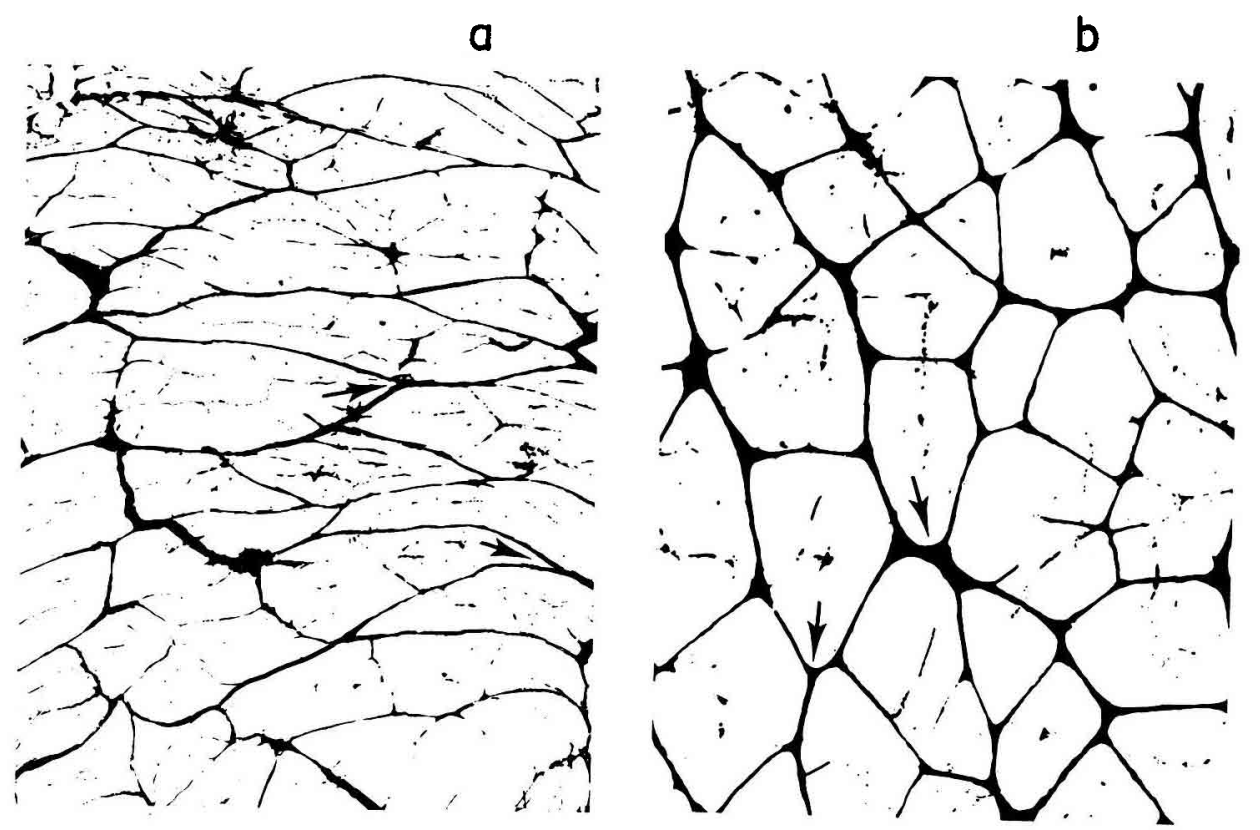

FIG. 15. - Exemple de forme de sommets. a : formes pointues; $b$ : formes arrondies ( $\times 5$ linéaire).

\section{Caractères des trames périmysiales secondaires.}

Nous rappelons que la trame périmysiale secondaire représente l'ensemble du périmysium que l'on trouve à l'intérieur de chaque faisceau principal, au sein de la trame périmysiale principale. L'aspect de la trame périmysiale secondaire peut différer légèrement d'une région à l'autre du muscle mais présente surtout une grande variété d'un type de muscle à l'autre. Les caractères descriptifs proposés pour décrire cette trame et en apprécier la variabilité sont les suivants : - degré de structuration (voir plus haut) ; - homogénéité de la trame ; - finesse de la trame ; - présence d'étoiles conjonctives.

- Homogénéité des trames périmysiales secondaires. - Quels que soient le degré de structuration, et le type de structuration (typique ou atypique), les trames périmysiales secondaires présentent un aspect général, plus ou moins homogène qui 

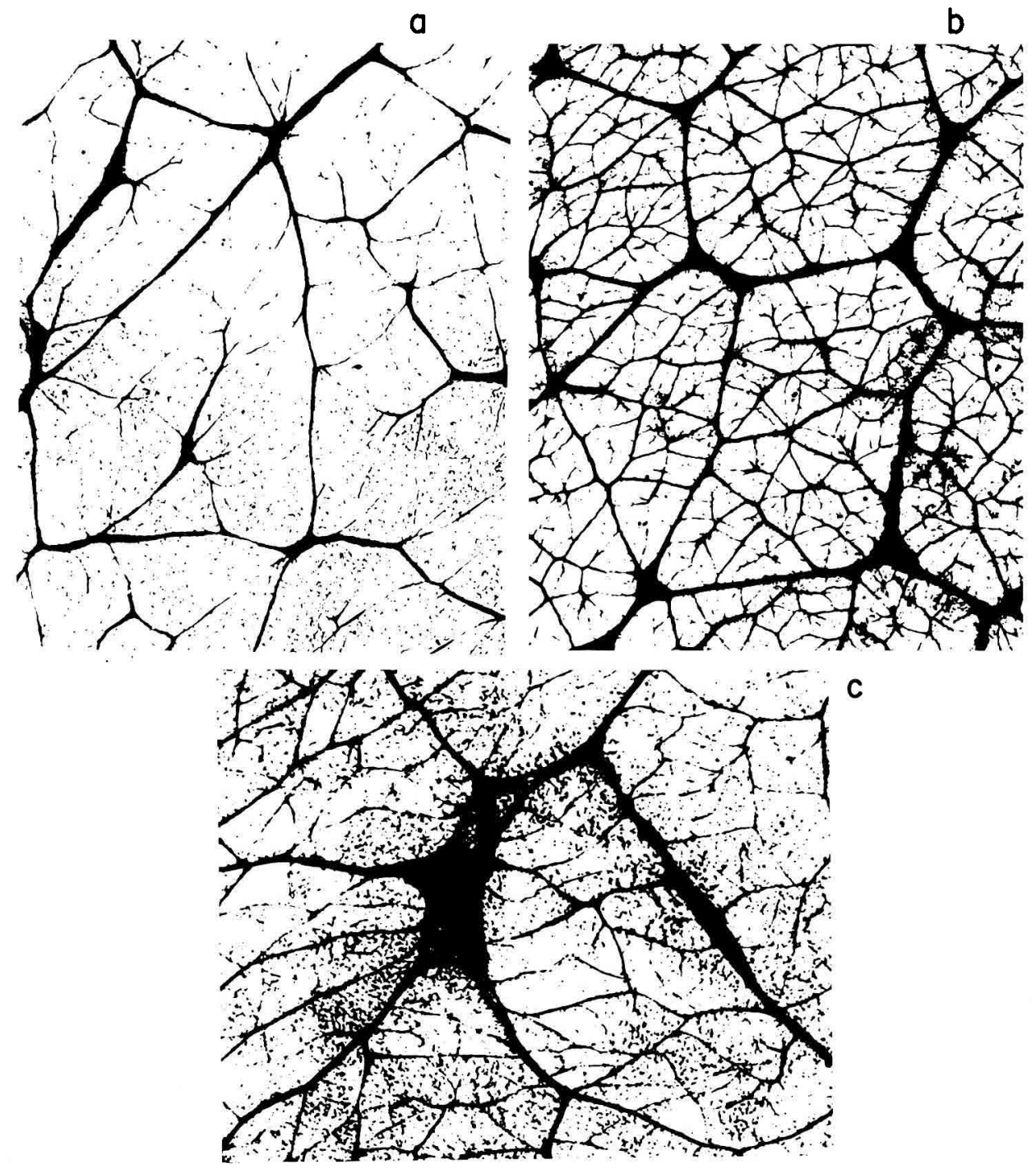

FIG. 16. - Trois types d'images présentant des nœuds, $a$ : noveux et petits ; $b$ : très noueux et moyens ; $c$ : peu noveux mais gros.

peut être traduit par le classement binaire proposé au tableau 1B. La figure 17 présente des exemples de trame périmysiale secondaire considérée comme homogène (17a) ou hétérogène $(17 b)$.

- Présence d'《étoiles » conjonctives. - On peut voir apparaître dans la trame périmysiale secondaire et, le plus souvent dans la partie centrale des faisceaux principaux, des noyaux de tissu conjonctif, d'importance variable, d'où rayonnent, en direction de la périphérie, des travées conjonctives interfasciculaires de différents 


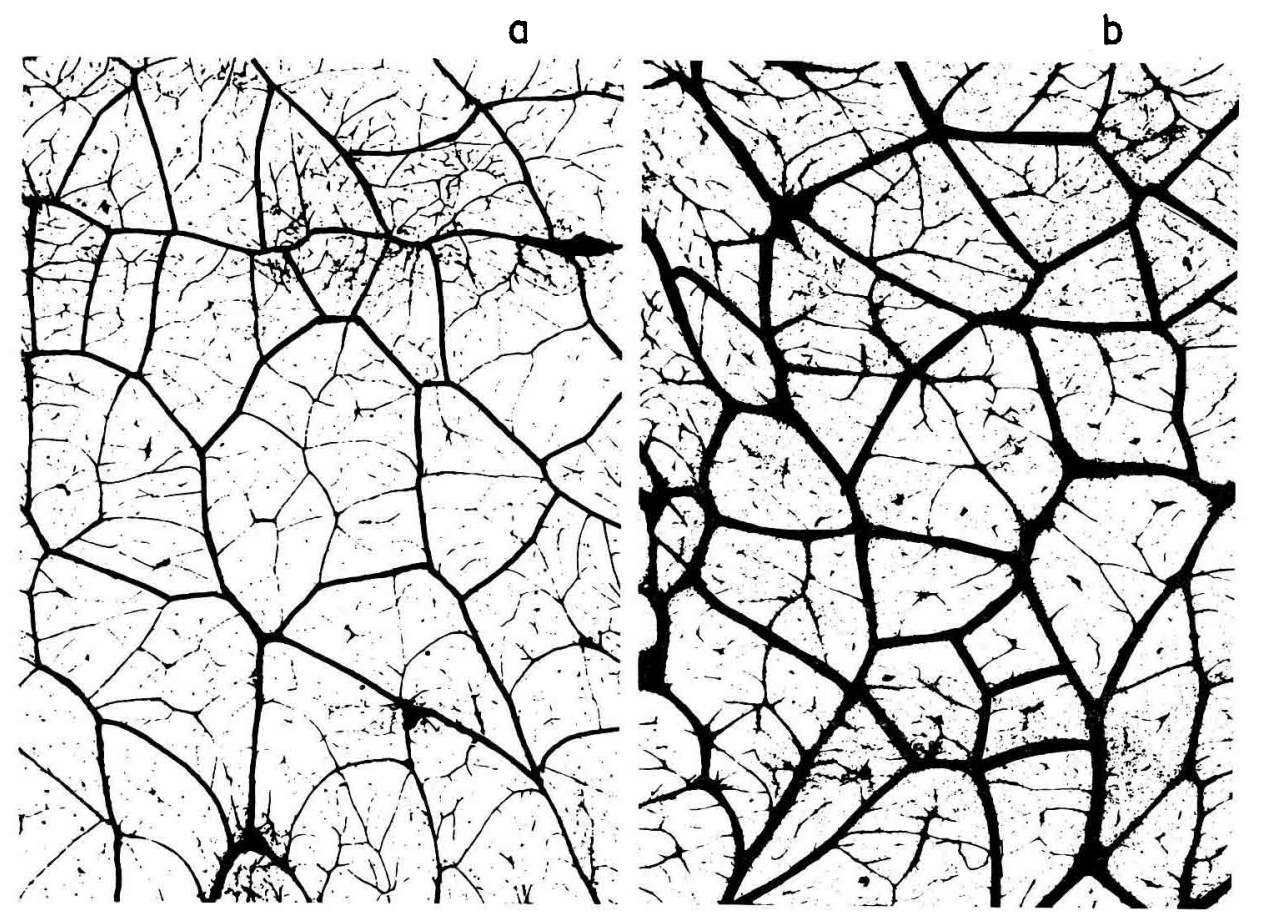

FIG. 17. - Exemple d'homogénéité de la trame secondaire. Pour ces deux photos les unités de structure ont été soulignées volontairement pour mieux apprécier les caractères : homogène et hétérogène.

ordres. Ces formations correspondent à ce que nous proposons d'appeler des "étoiles ». La figure 18 montre quelques exemples d'étoiles.

- Finesse des trames périmysiales secondaires. - A l'intérieur des faisceaux principaux la trame périmysiale secondaire apparaît sous forme d'un lacis plus ou moins dense ef épais, selon le degré de structuration, selon le nombre total des faisceaux primaires et selon l'épaisseur relative des parois interfasciculaires des différents ordres $(n-1, n-2, \ldots)$. L'impression de finesse que laisse à l'observateur l'examen des diverses parties du muscle est une sensation complexe qui résulte de la prise en compte, au moment du jugement, d'éléments variés comme l'importance de la trame périmysiale secondaire, sa densité, l'épaisseur des différentes parois interfasciculaires... Comme approche de l'estimation de ce caractère complexe il est suggéré de procéder au classement dans l'une des six catégories prévues pour ce caractère au tableau 1B (trames très fines à très épaisses). La figure 19A et B illustre différentes éventualités rencontrées dans la finesse de trames périmysiales secondaires dans différents types de muscles.

- Lames conjonctives intramusculaires. - Certains muscles présentent à l'intérieur de leur section transversale des épaississements très marqués, à certains niveaux de la trame principale du périmysium. Lorsque l'épaississement se produit sur une 


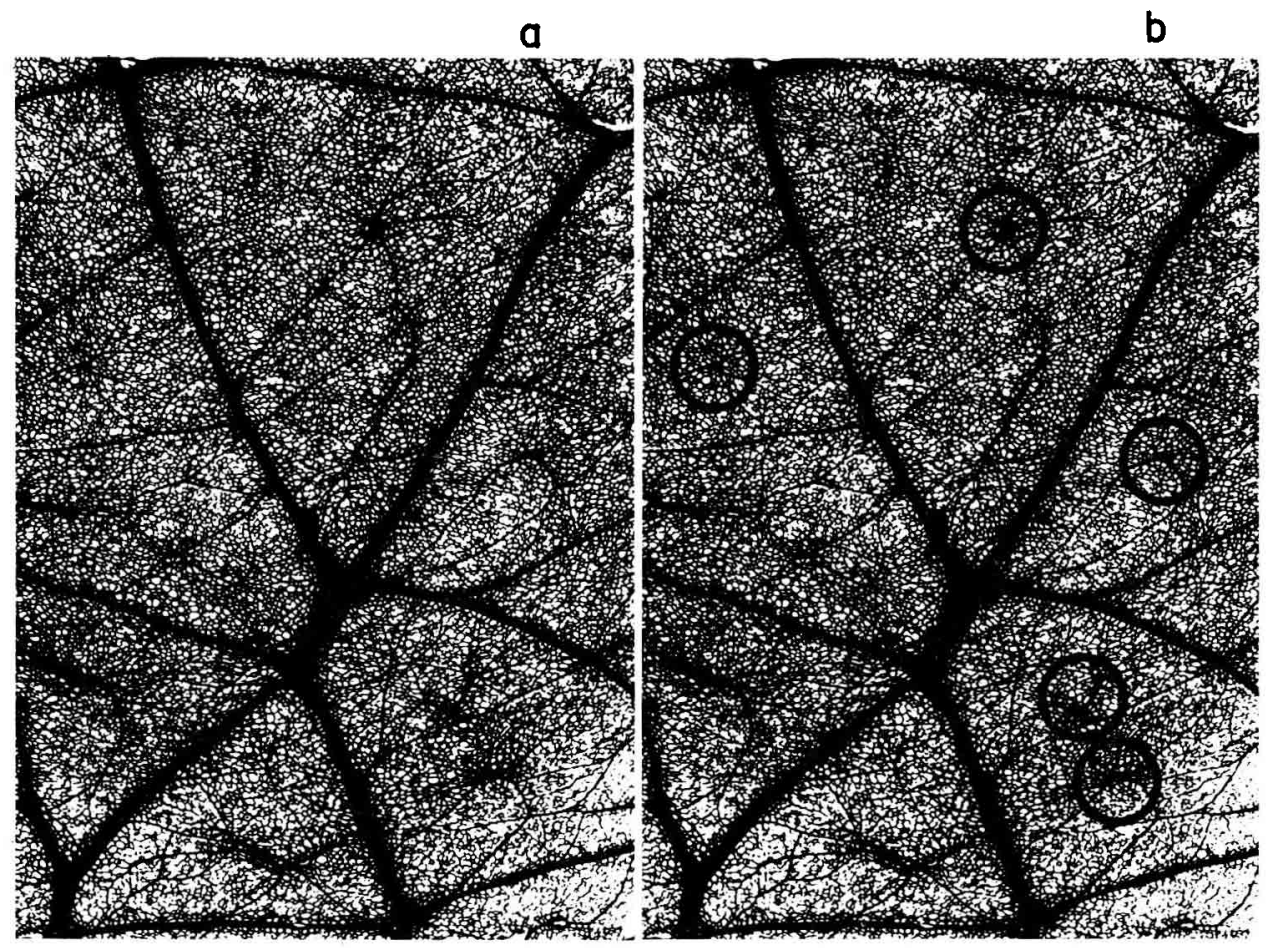

FIG. 18. - «Etailes conjonctives ». Les différentes étoiles dans la photo b ont été entourées pour faciliter leur caractérisation ( $\times 10$ linéaire).

certaine distance on peut parler de lame conjonctive intramusculaire. La figure 20 présente une coupe du $M$. semitendinosus de bovin présentant, à plusieurs endroits, de telles lames. Les lames conjonctives peuvent occuper une situation variable dans la section et connaître, en longueur, par rapport aux dimensions du muscle une importance variable. Réparition et importance peuvent se décrire de façon détaillée par rapport aux extrémités du muscle et à son axe principal. Pour un jugement rapide et global de ce caractère il est proposé d'utiliser les échelles de la table de description qui retiennent cinq éventualités pour la répartition et pour l'importance.

- Vaisseaux sanguins. - La trame conjonctive périmysiale est le support de l'ensemble des vaisseaux sanguins de toutes tailles qui irriguent et drainent le muscle. La majeure partie de ces vaisseaux sont de petites dimensions et ne sont décélables qu'à l'examen microscopique. Ne seront considérés ici comme participant à la caractérisation qualitative de la trame périmysiale que ceux des vaisseaux dont le diamètre est suffisamment grand pour qu'ils apparaissent à l'examen à grandissement limité pris comme référence dans cette étude $(x 10)$. On procède à leur dénombrement par comptage ainsi qu'à la description de leur localisation el à l'estimation de leur dimension. Pour ces caractères il est proposé de retenir l'échelle d'évaluation subjective du tableau $1 \mathrm{~B}$. 
2

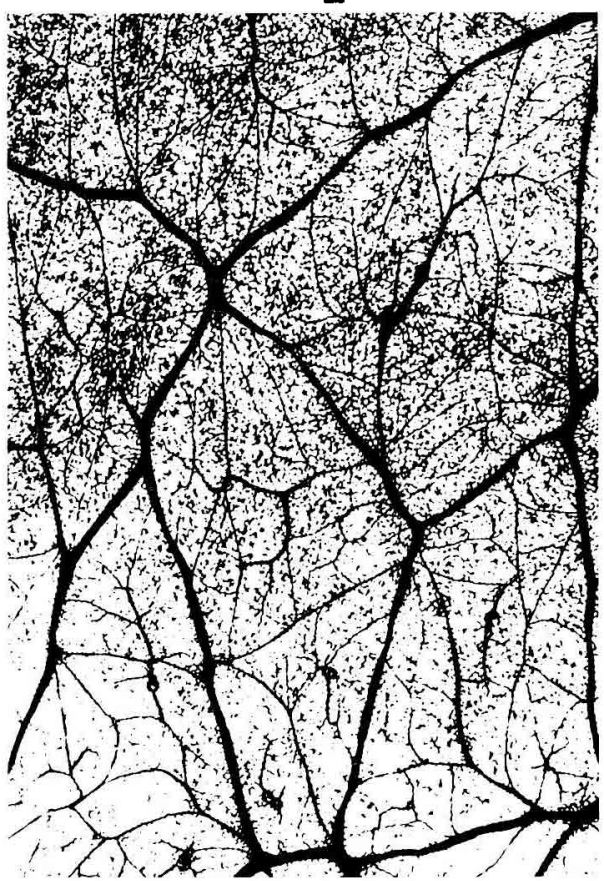

4

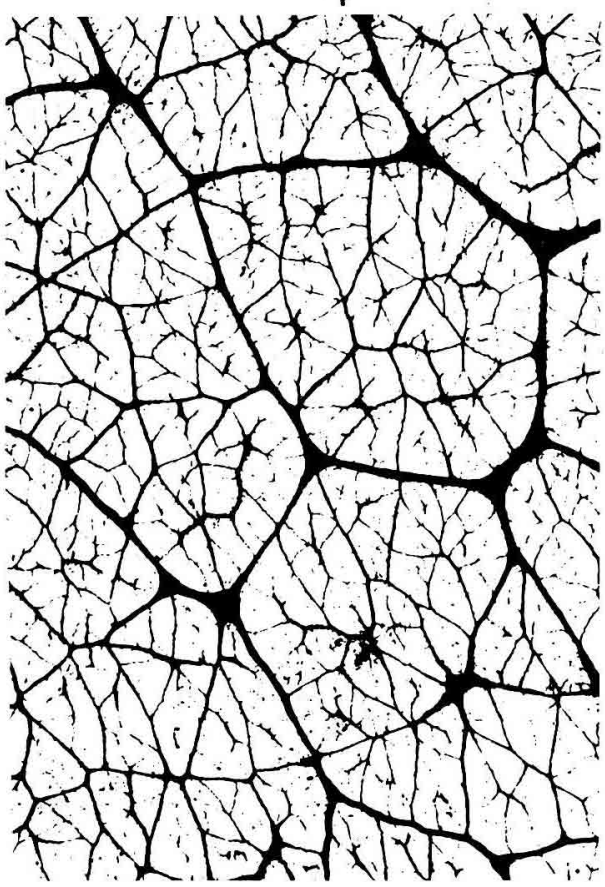

3

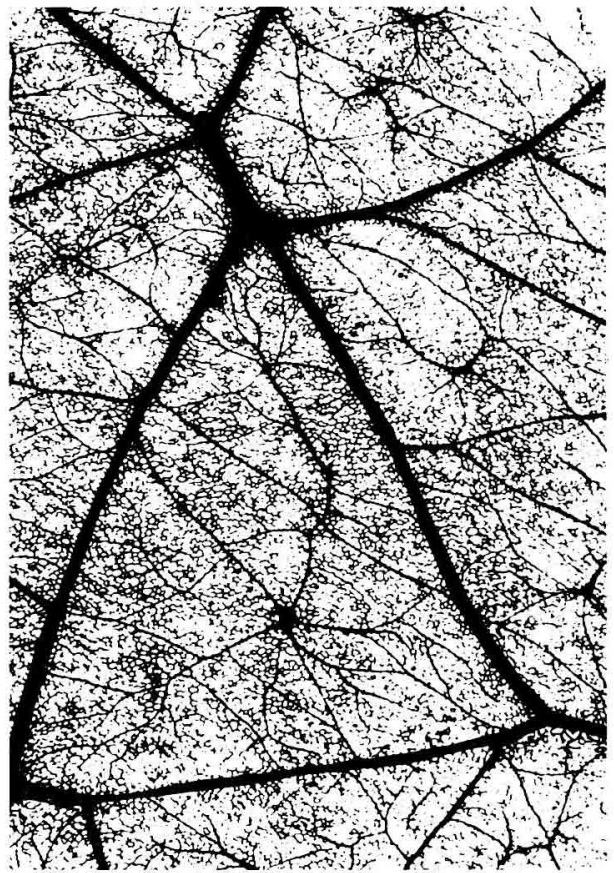

5

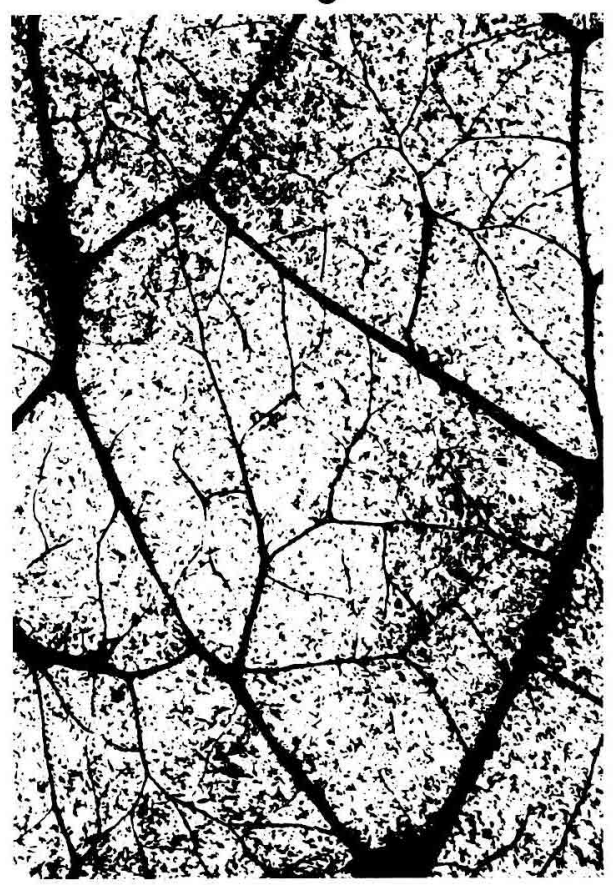

FIG. 19A. 
6

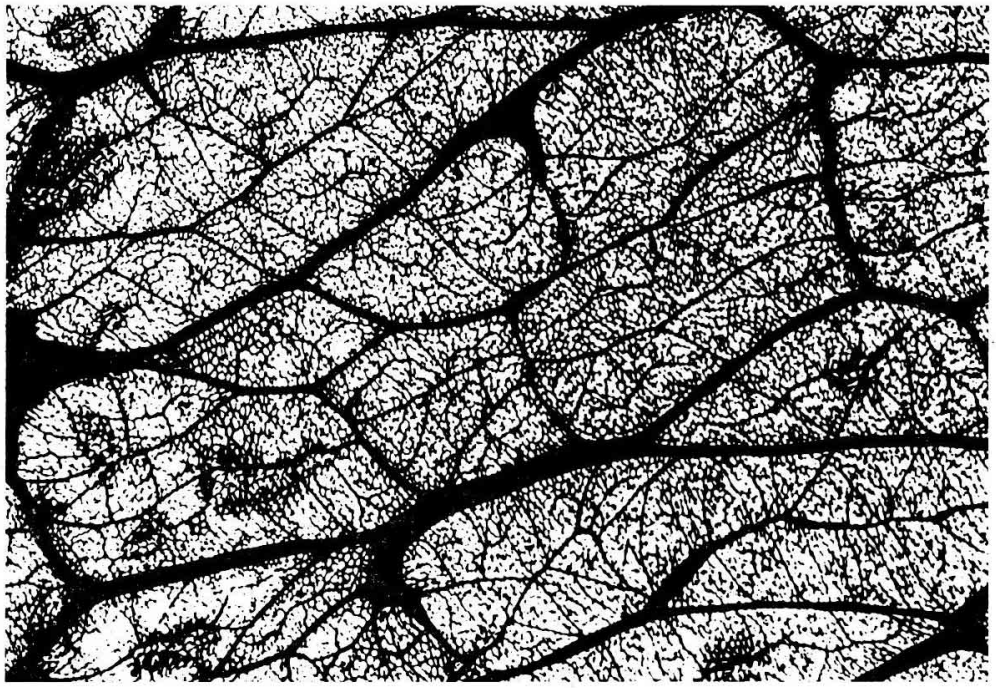

FIG. 19B.

FIG. 19A et B. - Finesse des trames périmysiales secondaires (voir tabl. 1B) ( $\times 10$ linéaire). La trame très fine (1) n'a pas été représentée car l'image n'aurait pu être obtenue sans un renforcement excessif du contraste.

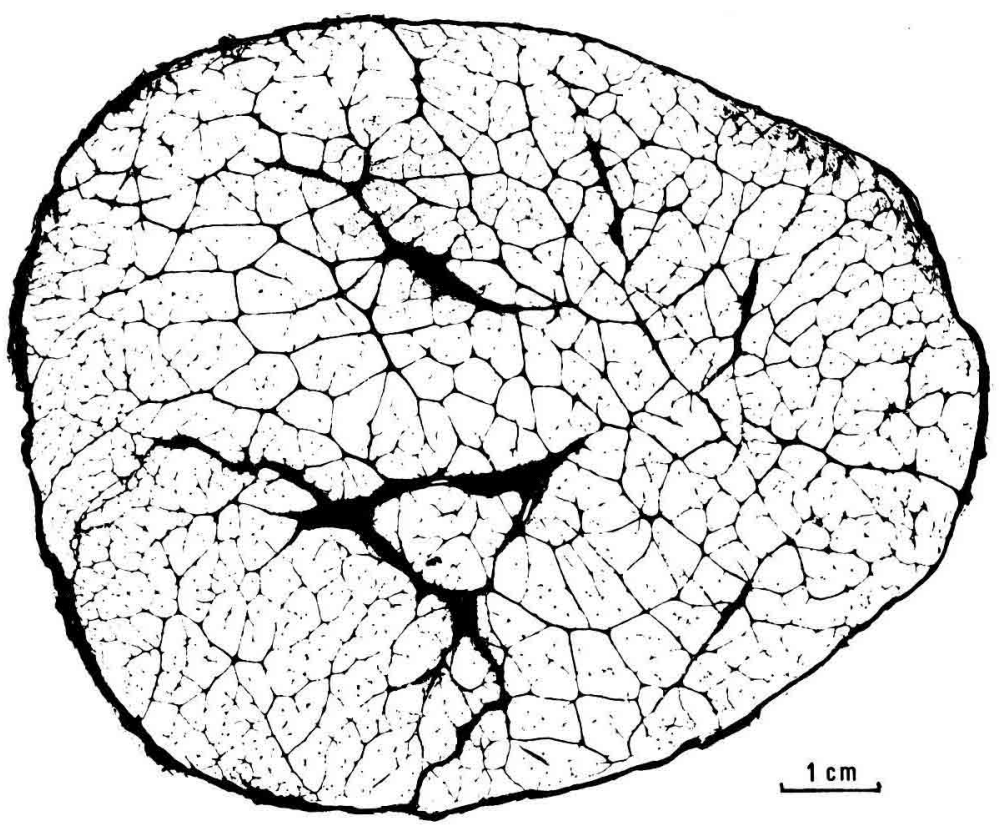

FIG. 20. - Les grosses travées noires représentent des lames conjonctives intramusculaires. 
- Taches conjonctives intramusculaires. - On peut rencontrer, dans certains muscles, des formations de tissu conjonctif qui se manifestent, dans la surface de section, sous forme de taches plus ou moins importantes tant en nombre qu'en taille. La présence de telles taches peut être mentionnée comme l'indique la table de description. La figure 21 donne des exemples de telles taches au sein d'un muscle.

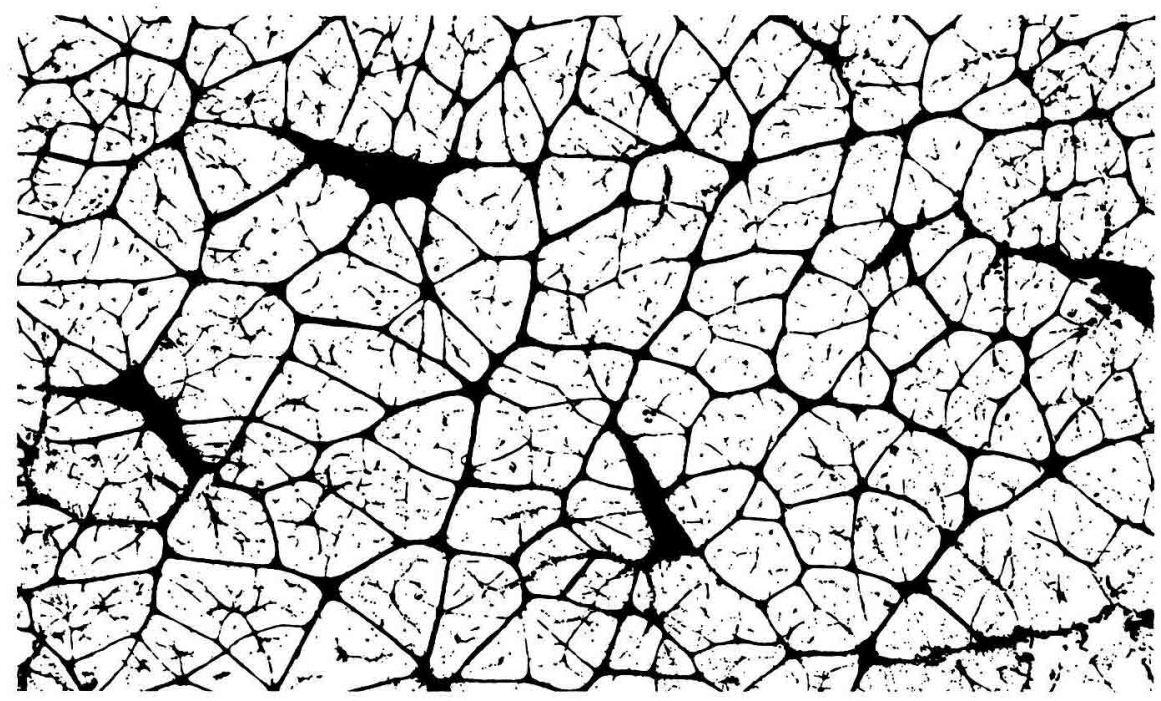

FIG. 21. - Taches conjonctives intramusculaires dans un M. semitendinosus de porcin ( $\times 5$ linéaire).

\section{Discussion et conclusion.}

La méthode proposée pour la description du périmysium fait appel à un grand nombre de caractères, soit qualifatifs, soit quantitatifs.

Une part importante de la description repose sur les caractères de la trame périmysiale générale dont la mise en évidence est le plus souvent très aisée, mais qui donne lieu quelquefois, dans certaines zones du muscle, à une appréciation subjective délicate et, par conséquent, laisse place à une certaine interprétation de la part de l'observateur.

D'autre part, il faut noter que la méthode proposée découle de l'observation d'échantillons fixés et traités histologiquement, ce qui peut, éventuellement, provoquer des artefacts de différente nature par rapport à la situation existant dans le matériel frais. A cet égard, les problèmes relevant de la coloration du tissu conjonctif peuvent être, en partie, résolus en supprimant la coloration de la coupe. Sur coupes non colorées et non montées mais simplement collées, la trame principale apparaît nettement, de même que l'essentiel de la trame secondaire. Toutefois la coloration reste indispensable pour une détermination précise du degré de structuration. 
L'application de la méthode proposée ici à des ensembles de muscles de bovins a permis, grâce à l'analyse multidimentionnelle, d'assurer entre eux une discrimination et une classification systématique, à partir d'un nombre assez restreint de caractères (Dumont ef al., 1977). Il apparaît donc qu'on peut reconnaître un muscle à l'examen de sa surface de section d'après les principaux traits de l'organisation de son périmysium.

L'emploi de cette méthode de description du périmysium doit permettre d'étudier les conséquences de la variation des facteurs biologiques tels l'espèce, le sexe, l'âge..., sur la structure morpho-anatomique du muscle. Ainsi, dans une étude de l'influence de l'âge et du poids sur la trame conjonctive, a-t-on pu mettre en évidence chez les bovins, qu'au cours de la croissance on assistait à une dilatation différentielle de la trame conjonctive existant à la naissance (Schmitt et Dumont, 1972) et que l'essentiel de la variation du réseau conjonctif était d'ordre quantitatif sans remise en cause du plan directeur fixant à la naissance l'organisation de la charpente périmysiale (Dumont et Schmitt, 1975). On a pu, également établir que dans le cas de l'hypertrophie musculaire manifestée par certains types génétiques de bovins, on enregistre une structure différente des muscles par suite d'un périmysium moins abondant et d'une trame beaucoup plus lâche (Dumont et Schmitt, 1973). Nous avons aussi abordé l'étude comparative de la trame conjonctive intramusculaire de différentes espèces (Schmitt, 1978).

II serait intéressant de développer des travaux sur les relałions existant entre les caractères de la trame conjonctive ef la nature du travail réalisé par les muscles, dans la mesure où l'on retient la proposition que la irame conjonctive d'un muscle donné est en rapport avec la fonction particulière de ce muscle. La méthode de description morpho-anatomique du périmysium que nous proposons permet une approche de ce problème.

II serait également important de relier la structure de la trame conjonctive et les caractères chimiques ef physiques du collagène que sont, soit son type biochimique, soit son degré de réticulation. On peut avancer, en effet, l'hypothèse que l'hétérogénéité micro-anatomique que révèle l'analyse descriptive de la trame s'accompagne d'une hétérogénéité dans les caractères physiques ou chimiques de ses constituants.

Sur un autre plan, l'analyse détaillée de la trame conjonctive doit permettre, en ce qui concerne la viande, de préciser l'influence relative des différents caractères du conjonctif sur les diverses composantes sensorielles de la dureté perçue par les consommateurs ainsi que sur les propriétés rhéologiques mesurées par des appareils appropriés. Une première approche de ce problème a été déjà envisagée dans le cas de la force de cisaillement de muscles de bovins mesurés à l'appareil de WarnerBratzler (Dumont et al., 1977).

Enfin, il serait intéressant d'étudier les relations existant entre les caractères de la trame conjonctive et l'importance des infiltrations graisseuses qui la pénètre (persillé) ef dont l'expérience pratique montre la grande variation d'un muscle à l'autre.

Les méthodes d'appréciation subjective proposées ici se révèlent, à l'usage, d'un emploi pratique pour des jugements rapides de série. Il est vraisemblable que pour de nombreux caractères, sinon pour tous, l'emploi d'appareils assurant des analyses dites automatiques des images permettra une objectivation des mesures, sinon une plus grande rapidité de leur exécution. On peut s'attendre également à ce que ces 
appareils permettent de mesurer d'autres caractères que ceux déterminés ici, par exemple des fréquences d'orientation des parois de la trame principale (Rowe, 1977).

Reçu en mars 1978.

Accepté en juillet 1978.

\section{Références}

BOURGELAT C., 1797. Eléments de l'art vétérinaire. Librairie vétérinaire de la citoyenne Huzard, Paris.

CASSENS R. G., 1971. Microscopic structure of animal tissue, meat and meat products, 11-77. In PRICE J. F., SCHWEIGERT B. S., The science of meat and meat products, 2 nd ed. W. H. Freeman and $C^{\circ}$, San Francisco.

DUMONT B., 1952. La tendreté de la viande. Ann. Zootech., 1, 71-95.

DUMONT B. L., SCHMITT O., 1969. Structure musculaire comparée de porcs Large White et Piétrain. Ann. Génét. Sél. anim., 1, 178-179.

DUMONT B. L., SCHMITT O., 1970. Anatomie microscopique comparée du tissu musculaire squelettique de porcs Large-Whiłe et Piétrain. Ann. Génét. Sél. anim., 2, 381-391.

DUMONT B. L., SCHMITT O., 1973. Conséquences de l'hypertrophie musculaire héréditaire sur la trame conjonctive du muscle de bovin. Ann. Génét. Sél. anim., 5, 499-506.

DUMONT B. L., SCHMITT O., 1975. Etude de la variation de la trame du tissu conjonctif musculaire chez le bovin (Bos taurus). Réun. Groupe "Développement ef croissance "INRA; INA, Paris-Grignon, 17-18 avril.

DUMONT B. L., LEFEBVRE J., SCHMITT O., BARBU S., 1977. Application de méthodes d'analyse multidimentionnelle à la différenciation des muscles sur la base de l'organisation de leur trame conjonctive. 10th eur. Meet. Stotist., Leuven (Belgium), 22-26th August 1977, 96.

FERNAND V. S. V., 1949. cité par Gould, 1973.

FORREST J. C., ABERLE E. D., HEDRICK H. B., JUDGE M. D., MERKEL R. A., 1975. Principles of meat science. W. H. Freeman and Co, San Francisco.

GOULD R. P., 1973. The microanatomy of muscle, 185-241. In BOURNE S. M. The structure and function of muscle, Vol. 2. Acad. Press, New-York.

ROWE R. W. D., 1977. The influence of the collagen fibre network of muscle on the compliance and tensile strength of muscle samples subjected to loads at right angles to the muscle fibre axis. Meat Sci., 1, 135-148.

SCHMITT O., DUMONT B. L., 1969. Méthodes d'analyse de la structure musculaire. Ann. Biol. anim. Bioch. Biophys., 9, 123-134.

SCHMITT O., DUMONT B. L., 1972. Conjonctif et croissance musculaire. Ann. Biol. anim. Bioch. Biophys., 12, 667-672.

SCHMITT O., 1978. Etude comparative de la trame conjonctive intramusculaire de différentes espèces d'animaux de boucherie (bovins, ovins, porcins ef caprins). Réun. Chercheurs en Viande, Paris, 13-14 déc. 1977, Ann. Technol. agric. (sous presse) (résumé). 